\title{
Self-organized criticality within fractional Lorenz scheme
}

\author{
Alexander I. Olemskoia,* Alexei V. Khomenko ${ }^{\mathrm{b}}$, \\ Dmitrii O. Kharchenko ${ }^{\mathrm{a}, 1}$ \\ ${ }^{a}$ Max-Planck-Institut für Physik komplexer Systeme, Nöthnitzer Strasse 38, \\ D-01187 Dresden, Germany \\ ${ }^{\mathrm{b}}$ Sumy State University, Rimskii-Korsakov St. 2, 40007 Sumy, Ukraine
}

Received 18 November 2002

\begin{abstract}
The theory of a flux steady-state related to avalanche formation is presented for the simplest model of a sand pile within the framework of the Lorenz approach. The stationary values of sand velocity and sand pile slope are derived as functions of a control parameter (driven sand pile slope). The additive noise of above values are introduced for building a phase diagram, where the noise intensities determine both avalanche and non-avalanche domains, as well as mixed one. Corresponding to the SOC regime, the last domain is crucial to affect of the noise intensity of the vertical component of sand velocity and especially sand pile slope. To address to a self-similar behavior, a fractional feedback is used as an efficient ingredient of the modified Lorenz system. In the spirit of Edwards paradigm, an effective thermodynamics is introduced to determine a distribution over an avalanche ensemble with negative temperature. Steady-state behavior of the moving grains number, as well as non-extensive values of entropy and energy is studied in detail. The power law distribution over the avalanche size is described within a fractional Lorenz scheme, where the energy noise plays a crucial role. This distribution is shown to be a solution of both fractional and nonlinear Fokker-Planck equation. As a result, we obtain new relations between the exponent of the size distribution, fractal dimension of phase space, characteristic exponent of multiplicative noise, number of governing equations, dynamical exponents and non-extensivity parameter.

(c) 2003 Elsevier Science B.V. All rights reserved.
\end{abstract}

${ }^{*}$ Corresponding author. Sumy State University, Rimskii-Korsakov St. 2, Sumy 40007, Ukraine.

E-mail addresses: olemskoi@sumdu.edu.ua (A.I. Olemskoi), khom@phe.sumdu.edu.ua (A.V. Khomenko), dikh@sumdu.edu.ua (D.O. Kharchenko).

${ }^{1}$ Present address: Sumy State University, Rimskii-Korsakov St. 2, 40007 Sumy, Ukraine. 
PACS: 05.45.-a; 05.65.+b; 45.70.-n; 64.60.Ht

Keywords: Sand velocity and slope; Lorenz equations; Noise

\section{Introduction}

In recent years considerable study has been given to the theory of self-organized criticality (SOC) that explains spontaneous (avalanche-type) dynamics, unlike the typical phase transitions that occur only when a control parameter is driven to a critical value $[1,2]$. A main feature of the systems displaying SOC is their self-similarity that leads to a power-law distribution over avalanche sizes. Respectively, in most cases, SOC models are studied by making use of the scaling-type arguments supplemented with extensive computer simulations (see Ref. [3]). In contrast, we put forward an analytical approach, which is able to describe both the process of a single avalanche formation and the behavior of a whole avalanche ensemble in a phenomenological manner.

The SOC behavior appears in a vast variety of systems, such as real sand pile (ensemble of grains of sand moving along increasingly tilted surface) [4-7], intermittency in biological evolution [8], earthquakes and forest-fires, depinning transitions in random medium and so on (see Ref. [9]). Among the above models, the sandpile is the simplest and both analytically [10,11] and numerically [12-14] most widely studied. In the analytical treatments a variety of field theory approaches should be noticed. Among them, the field scheme [15], based on a nonlinear diffusion equation that has failed to account for the main feature of self-organizing systems - the self-consistent character of avalanche dynamics. The obvious reason is that using an one-parameter approach does not take into account a feedback between the open subsystem and the environment, that are related to order and control parameters, respectively (see also the criticism in Refs. [5,6]). A much more substantial picture is given within two-parameter approaches $[5-7,16]$ that use both fundamental fields: gauge ones related to hydrodynamical modes type of sand pile height and material fields as a number of moving sand grains (avalanche size). The mean-field approximation shows that the self-similar regime of the sand pile dynamics is relevant for subcritical behavior, where a characteristic time for the variation of the order parameter is much larger than that of the control parameter. Moreover, the latter follows the former adiabatically. Adiabatic behavior of this type is inherent in the usual regime of a system evolving in course of phase transitions [17] and jammed motion of vehicles [18], so that an adiabatic approach will be taken as basis of our consideration.

Perfect treatment of the SOC has been achieved within three-parameter approach based on the Reggeon field theory that uses the density of active sites $\rho_{a}$ as order parameter and the conserved field of the energy density $\zeta$ as the control parameter $[19,20]$. Along this line, the SOC regime appears as a result of competition between a rate of the energy input $h>0$ and a dissipation rate $\varepsilon$. The considered system behaves in quite a different manner when the energy is fixed, i.e., $h=\varepsilon=0$, and total energy is conserved, and for a driven sandpile, when $h \rightarrow 0^{+}, \varepsilon \rightarrow 0$ under the stationarity condition $\varepsilon>h$. The first case can be reduced $[19,20]$ to the picture of supercritical regime, 
where a non-homogeneity of the initial energy distribution results in a non-Markovian term and space-dependent parameters. At dimensions above the critical value $d_{c}=4$, this case is found to be identical to the simplest Landau picture with $\rho_{a} \sim\left(\zeta-\zeta_{c}\right)$ in the active stationary state $\left(\zeta>\zeta_{c}\right)$ and $\rho_{a}=0$ in the absorbing configuration $\left(\zeta<\zeta_{c}\right)$. A fundamentally different picture appears in the case of a driven sandpile, where due to external input $h \rightarrow 0^{+}$, the energy density is no longer an independent field that can be reduced to the critical value $\zeta_{c}$. In this case, the average magnitude of the density of active sites is equal to $\left\langle\rho_{a}\right\rangle=h / \varepsilon$, so that the susceptibility $\chi \equiv\left\langle\partial \rho_{a} / \partial h\right\rangle$ turns out to be $\chi=\varepsilon^{-1}$. As a consequence, a response function behaves as $\chi(r) \sim r^{2-d} \mathrm{e}^{-r / \xi}$ at large distances $r$, where $d$ is a space dimension, and $\xi \sim \varepsilon^{-1 / 2}$ is a correlation length that is a scale for the system size $L \sim \varepsilon^{-1 / \mu}$. It is remarkable that such a mean-field-type behavior is caused solely by a stationary condition and a translational invariance [20]. Respectively, a set of basic critical exponents governing the scaling of avalanche formation reads [19]: $\beta=\gamma=\delta=1, \mu=2, v=\frac{1}{2}$ and $\eta=0$. On the other hand, scaling relations accompanied by the equality of the susceptibility and the mean size of avalanche lead to the following expressions

$$
\tau=1+\frac{z}{D}, \quad \tau=2\left(1-\frac{1}{D}\right), \quad D=\frac{\mu}{\sigma}
$$

for the exponents of the avalanche size distribution

$$
P(s, \varepsilon)=s^{-\tau} \mathscr{P}(x), \quad x \equiv s / s_{c}, \quad s_{c} \sim \varepsilon^{-1 / \sigma},
$$

where a critical size $s_{c}$ is connected to the system size $L \sim \xi$ and a characteristic time $t_{c} \sim L^{z}$ as follows $s_{c} \sim L^{D} \sim t_{c}^{D / z}$ (exponents $D=\mu / \sigma$ and $z$ are fractal dimension, and dynamical exponent related to a critical avalanche). According to Ref. [19], the mean-field magnitudes of the above exponents are given by: $\tau=\frac{3}{2}, \sigma=\frac{1}{2}, D=4$ and $z=2$.

In accordance with the standard approach [21], we will use as the gauge, as the material fields. The former are reduced to velocity components and sand pile slope considered while studying a single avalanche formation, whereas the latter are reduced to a number of moving sand grains at examination of a distribution over avalanche sizes. Section 2 contains the self-consistent theory of the flux steady-state developed along the first direction. It enables us to treat the problem of a single avalanche formation on the basis of the unified analytical approach that is relevant for the case of fixed energy in Ref. [20]. In Section 3 we take into account additive noises of the sand velocity components and sand pile slope. By this, an increase of the noise intensities causes avalanche emergence even in non-driven systems, where the control parameter noise plays a crucial role. A fluctuational regime of this type corresponds to the case $h \rightarrow 0^{+}$[20], where a distribution of the order parameter appears in an algebraic form with integer exponent. In order to not being restricted to such a particular case, in Section 4 we introduce a unified Lorenz system with a fractional feedback. This assumption allows us to describe a subcritical regime of the avalanche formation in natural manner. The above generalization puts forward basis of Section 5 devoted to consideration of avalanche ensemble. Following famous Edwards paradigm [22,23], an effective scheme addressed to non-extensive thermodynamics [24] is proposed to determine a time-dependent distribution over energies of moving sand grains. 
To generalize the Edwards scheme to non-stationary non-extensive systems, we use the fractional Lorenz system, where the avalanche size plays a role of the order parameter, non-extensive complexity is reduced to the conjugate field and the non-conserved energy of the moving grains is the control parameter. Within the framework of this approach, the phase diagram is calculated to define the different domains of system behavior as a function of noise intensities of the above values. As a result, we arrive at a natural conclusion that the power-law distribution (2) inherent in the SOC regime is caused by noise of the energy.

In Section 6 we show that this distribution is the solution of both nonlinear FokkerPlanck equation, that appears in the description of non-extensive systems [24], and fractional Fokker-Planck equation inherent in Lévy-type processes characterized by a dynamical exponent $z$ [25]. As a result, we obtain new relations between the exponent $\tau$ of the distribution (2), fractal dimension $D$ of phase space, characteristic exponent of multiplicative noise, a number of governing equations needed to present self-consistent behavior in SOC regime, dynamical exponent $z$ and Tsallis nonextensivity parameter $q$.

Appendix A contains the basic properties of fractional integral and derivative, as well as Jackson derivative.

\section{Noiseless avalanche formation}

Within the framework of the simplest model of a real sand pile, its surface at given time $t$ is defined by dependence $y=y(t, x)$. The flow of sand can locally be described in terms of three quantities: the horizontal and vertical components of the sand velocity, $\dot{x} \equiv \partial x / \partial t, \dot{y} \equiv \partial y / \partial t$, and the surface slope $y^{\prime} \equiv \partial y / \partial x$. The key point of our approach is that the above degrees of freedom are assumed to be of a dissipative type, so that, when they are not coupled, their relaxation to the steady state is governed by the Debye-type equations:

$$
\begin{aligned}
& \frac{\mathrm{d} \dot{x}}{\mathrm{~d} t}=-\frac{\dot{x}}{\tau_{x}}, \\
& \frac{\mathrm{d} \dot{y}}{\mathrm{~d} t}=-\frac{\dot{y}}{\tau_{y}^{(0)}}, \\
& \frac{\mathrm{d} y^{\prime}}{\mathrm{d} t}=\frac{y_{0}^{\prime}-y^{\prime}}{\tau_{S}},
\end{aligned}
$$

where $\tau_{x}, \tau_{y}^{(0)}$ and $\tau_{S}$ are the relaxation times of the velocity components and the slope, respectively. Eqs. (3)-(5) imply that the sand rests in the stationary state, $\dot{x}=\dot{y}=0$, and the equilibrium slope $y^{\prime}=y_{0}^{\prime} \neq 0$ plays the role of a control parameter.

Since the motion of a sand grain along different directions is not independent of each other, Eq. (3) should be changed by adding the term $f=\dot{y} / \gamma$, that describes a liquid friction force along the $y$-axis ( $\gamma$ being the kinetic coefficient). Then, we have

$$
\tau_{x} \ddot{x}=-\dot{x}+a^{-1} \dot{y}
$$


where $a \equiv \gamma / \tau_{x}$. Note that, owing to the diffusion equation $\dot{y}=D y^{\prime \prime}$ ( $D$ is a diffusion coefficient), the friction force seems to be driven by the curvature of the sand pile surface

$$
f=(D / \gamma) y^{\prime \prime} .
$$

In the stationary state, when $\ddot{x}=0$, the solution of Eq. (6) defines the tangent line $y=a x+$ const, so that the friction force $f=\tau_{x}^{-1} \dot{x}$ is proportional to the horizontal component of the sand velocity. Taking into consideration the relation (7) and using the chain rule $\mathrm{d} y^{\prime} / \mathrm{d} t=\dot{y}^{\prime}+y^{\prime \prime} \dot{x}$, Eq. (5) leads to the equation of motion for the slope

$$
\tau_{S} \dot{y}^{\prime}=\left(y_{0}^{\prime}-y^{\prime}\right)-\left(\tau_{S} / D\right) \dot{y} \dot{x} .
$$

In a similar manner, the equation for the vertical component of the velocity can be deduced

$$
\tau_{y} \ddot{y}=-\dot{y}+\frac{\tau_{y}}{\tau_{x}} y^{\prime} \dot{x}, \quad \frac{1}{\tau_{y}} \equiv \frac{1}{\tau_{y}^{(0)}}\left(1+\frac{y_{0}^{\prime}}{a} \frac{\tau_{y}^{(0)}}{\tau_{x}}\right) .
$$

Note that the higher order terms are disregarded in Eq. (9) and the renormalized relaxation time $\tau_{y}$ depending on the stationary slope $y_{0}^{\prime}$ is introduced.

Eqs. (6), (8) and (9) constitute the basis for self-consistent description of the sand flow on the surface with the slope $y^{\prime}$ driven by the control parameter $y_{0}^{\prime}$. The distinguishing feature of these equations is that nonlinear terms, that enter Eqs. (8) and (9), are of opposite signs, while Eq. (6) is linear. Physically, the latter means that on the early stage the avalanche begins to move along the tangent $y=a x+$ const. The negative sign of the last term in Eq. (8) can be regarded as a manifestation of Le Chatelier principle, i.e., since the slope's increase results in the formation of an avalanche, the velocity components $\dot{x}$ and $\dot{y}$ tend to impede the growth of the slope. The positive feedback of $\dot{x}$ and $y^{\prime}$ on $\dot{y}$ in Eq. (9) plays a fundamental role in the problem. As we will show later, that it is precisely the reason for the self-organization that causes the avalanche generation.

After a suitable rescaling, Eqs. (6), (8) and (9) can be rewritten in the form of the well-known Lorenz system

$$
\begin{aligned}
& \dot{u}=-u+v, \\
& \varepsilon \dot{v}=-v+u S, \\
& \delta \dot{S}=\left(S_{0}-S\right)-u v,
\end{aligned}
$$

where $u \equiv\left(\tau_{y} / \tau_{x}\right)^{1 / 2}\left(\tau_{S} / D\right)^{1 / 2} \dot{x}, v \equiv\left(\tau_{y} / \tau_{x}\right)^{1 / 2}\left(\tau_{S} / D\right)^{1 / 2} \dot{y} / a$, and $S \equiv\left(\tau_{y} / \tau_{x}\right) y^{\prime} / a$ are the dimensionless velocity components and the slope, respectively; $\varepsilon \equiv \tau_{y} / \tau_{x}, \delta \equiv \tau_{S} / \tau_{x}$ and the dot now stands for the derivative with respect to the dimensionless time $t / \tau_{x}$. In the general case, system (10)-(12) cannot be solved analytically, but in the simplest case, where $\varepsilon \ll 1$ and $\delta \ll 1$, the vertical velocity $v$ and the slope $S$ can be eliminated by making use of the adiabatic approximation that implies the neglection of the left-hand side of Eqs. (11) and (12). As a result, the dependencies of $S$ and $v$ on the horizontal velocity $u$ are given by

$$
S=\frac{S_{0}}{1+u^{2}}, \quad v=\frac{S_{0} u}{1+u^{2}} .
$$


Note that, under the assumption that $u$ is within the physically meaningful range between 0 and 1 , the slope $S$ is a monotonically decreasing function of $u$, whereas the velocity $v$ increases with $u$ (at $u>1$ we have $\mathrm{d} v / \mathrm{d} u<0$ and the flow of the sand becomes unstable).

Substitution of the second Eq. (13) into Eq. (10) yields the Landau-Khalatnikov equation

$$
\dot{u}=-\frac{\partial E}{\partial u}
$$

with the kinetic energy

$$
E=\frac{1}{2} u^{2}-\frac{1}{2} S_{0} \ln \left(1+u^{2}\right) .
$$

For $S_{0}<1, E$ is a monotonically increasing function of $u$ and the only stationary value of $u$ equals zero, $u_{0}=0$, so that there are no avalanches in this case. Obviously, such steady-state relevant for absorbing configuration studied in Ref. [20]. If the slope $S_{0}$ exceeds the critical value, $S_{c}=1$, the kinetic energy takes its minimum with the non-zero steady-state velocity components $u_{e}=v_{e}=\left(S_{0}-1\right)^{1 / 2}$ and the slope $S_{e}=1$.

The above scenario represents a supercritical regime of an avalanche formation and is related to a second-order phase transition [16]. The latter can be easily seen from the expansion of the kinetic energy (15) in a power series of $u^{2} \ll 1$. So the critical exponents $\gamma, \delta, v$ are identical to those obtained within the framework of the mean-field theory [19]. However, the magnitude $\beta=\frac{1}{2}$ is twice as little because our order parameter (the velocity) is not reduced to the same (the number of active sites) in theory [19].

It is a drawback of the outlined approach that it fails to account for the subcritical regime of the self-organization. That is the reason for the appearance of avalanches and analogous to the first-order phase transition rather than the second-order one. So one has to modify the above theory by assuming that the effective relaxation time $\tau_{x}(u)$ increases with the velocity $u$ from a value $\tau_{x}(1+m)^{-1}, m>0$ to $\tau_{x}$ [17]. The simplest two-parameter approximation is

$$
\frac{\tau_{x}}{\tau_{x}(u)}=1+\frac{m}{1+\left(u / u_{0}\right)^{2}}
$$

where $0<u_{0}<1$. The expression for the kinetic energy (15) then changes by adding the term

$$
\Delta E=\frac{m}{2} u_{0}^{2} \ln \left[1+\left(\frac{u}{u_{0}}\right)^{2}\right]
$$

and the stationary values of $u$ are following:

$$
\begin{aligned}
& u_{e}^{m}=u_{00}\left\{1 \mp\left[1+u_{0}^{2} u_{00}^{-4}\left(S_{0}-S_{c}\right)\right]^{1 / 2}\right\}^{1 / 2}, \\
& 2 u_{00}^{2} \equiv\left(S_{0}-1\right)+S_{c} u_{0}^{2}, \quad S_{c} \equiv 1+m .
\end{aligned}
$$

The upper sign on the right-hand side of Eq. (18) meets the value of the unstable state $u^{m}$, where the kinetic energy $E+\Delta E$ has a maximum, the lower one corresponds to the stable state $u_{e}$. The corresponding values of the stationary slope are equal

$$
S^{ \pm}=\frac{1+u_{00}^{2} \pm \sqrt{\left(1+u_{00}^{2}\right)^{2}-\left(1-u_{0}^{2}\right) S_{0}}}{1-u_{0}^{2}} .
$$



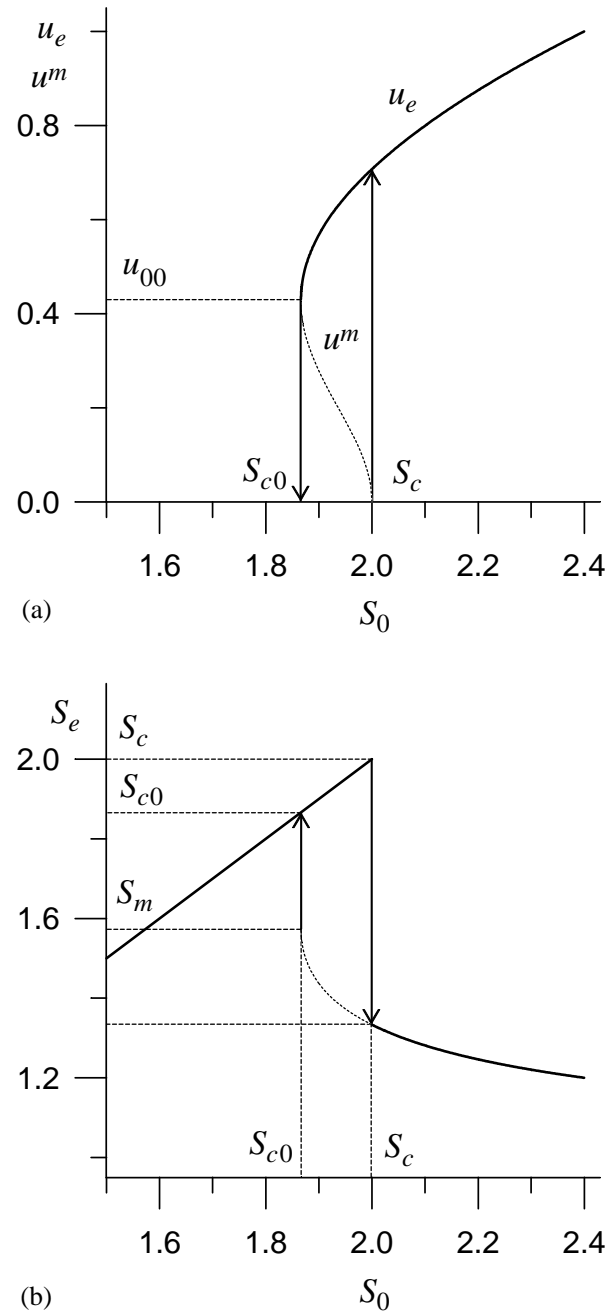

Fig. 1. The $S_{0}$-dependencies of (a) the velocities $u_{e}, u^{m}$, and (b) the equilibrium slope $S_{e}$. The arrows indicate the hysteresis loop.

The larger value $S^{+}$meets the unstable state and smoothly increases from the quantity

$$
S_{m}=1+u_{0} \sqrt{m /\left(1-u_{0}^{2}\right)}
$$

at the parameter $S_{0}=S_{c 0}$ with

$$
S_{c 0}=\left(1-u_{0}^{2}\right) S_{m}^{2}
$$

to the marginal value $S_{c}=1+m$ at $S_{0}=S_{c}$. The $S_{0}$-dependence of $u_{e}, u^{m}$, and $S_{e}$ are presented in Fig. 1. As shown in Fig. 1a, under the adiabatic condition $\tau_{S} \ll \tau_{x}$ is met and the parameter $S_{0}$ slowly increases being below $S_{c}\left(S_{0} \leqslant S_{c}\right)$, no avalanches can 
form. At the point $S_{0}=S_{c}$ the velocity $u_{e}$ jumps upward to the value $\sqrt{2} u_{00}$ and its further smooth increase is determined by Eq. (18). If the parameter $S_{0}$ then decreases, the velocity $u_{e}$ continuously decreases to the point, where $S_{0}=S_{c 0}$ and $u_{e}=u_{00}$. At this point the velocity instantaneously falls to zero. Referring to Fig. 1b, the stationary slope $S_{e}$ shows a linear increase from 0 to $S_{c}$ with the parameter $S_{0}$ being in the same interval and, after the jump down to the value $\left(1-u_{0}^{2}\right)^{-1}$ at $S_{0}=S_{c}, S_{e}$ smoothly decays to 1 at $S_{0} \gg S_{c}$. When the parameter $S_{0}$ then decreases from $S_{c}$ to $S_{c 0}$, the slope grows. When the point $S_{c 0},(21)$ is reached, the avalanche stops, so that the slope undergoes the jump from $S_{m}$, (20) up to $S_{c 0}$. For $S_{0}<S_{c 0}$, again the parameter $S_{e}$ does not differ from $S_{0}$. Note that this subcritical regime is realized, provided the parameter $m$, that enters the dispersion law (16), is greater than

$$
m_{\min }=\frac{u_{0}^{2}}{1-u_{0}^{2}} .
$$

According to the picture described, the avalanche generation is characterized by the well pronounced hysteresis, when the grains of sand initially being at rest begin to move downhill only if the slope of the surface exceeds its limiting value $S_{c}=1+m$, whereas the slope $S_{c 0}$ needed to stop the avalanche is less than $S_{c}$ (see Eqs. (20) and (21)). This is the case in the limit $\tau_{S} / \tau_{x} \rightarrow 0$ and the hysteresis loop shrinks with the growth of the adiabaticity parameter $\delta \equiv \tau_{S} / \tau_{x}$. In addition to the smallness of $\delta$, the adiabatic approximation implies also, that the ratio $\tau_{y} / \tau_{x} \equiv \varepsilon$ is small. In contrast to the former assumption, the latter does not seem to be realistic for the considered system, where in general $\tau_{y} \approx \tau_{x}$. Thus, it is of interest to study to what extent the finite value of $\varepsilon$ could change the results.

Owing to the condition $\delta \ll 1$, Eq. (12) is still algebraic and $S$ can be expressed in terms of $u$ and $v$. As a result, we derive a system of two nonlinear differential equations that can be studied with the phase portrait method [17]. The phase portraits for various values of $\varepsilon$ are displayed in Fig. 2, where the node point $O$ represents the stationary state and the saddle point $S$ is related to the maximum of the kinetic energy. As is obvious from Fig. 2, independent of $\varepsilon$, there is a universal section that attracts all phase trajectories and its structure seems to be almost insensitive to changes in $\varepsilon$. An analysis of time dependencies $v(t)$ and $u(t)$ reveals that the velocity components slow down appreciably in this section in comparison to the remaining parts of trajectories that are almost rectilinear (it is not difficult to see that this effect is caused by the smallness of the parameter $\delta$ ). Since most of the time the system is in vicinity of this universal section, we arrive at the conclusion that finite values of $\varepsilon$ qualitatively do not affect the results obtained in the adiabatic approximation.

\section{Noise influence on avalanche formation}

We now focus on the affect of additive noises of the velocity components $u$, $v$, and the slope $S$. With this aim, we should add to right-hand side of Eqs. (10)-(12) the stochastic terms $I_{u}^{1 / 2} \xi, I_{v}^{1 / 2} \xi, I_{S}^{1 / 2} \xi$ (here the noise intensities $I_{u}, I_{v}, I_{S}$ are measured in units $\left(\tau_{x} / \tau_{y}\right)\left(D / \tau_{S}\right), a^{2}\left(\tau_{x} / \tau_{y}\right)\left(D / \tau_{S}\right), a^{2}\left(\tau_{x} / \tau_{y}\right)$, correspondingly, and $\xi(t)$ is 


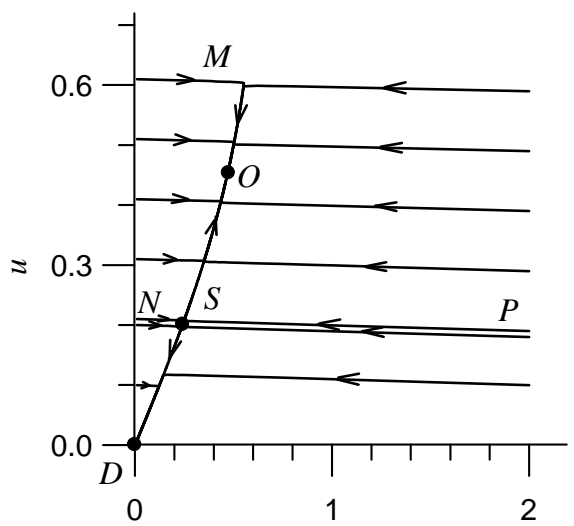

(a)

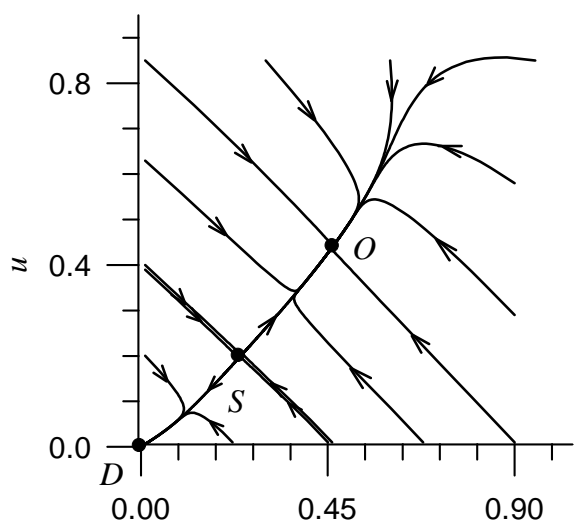

(b)

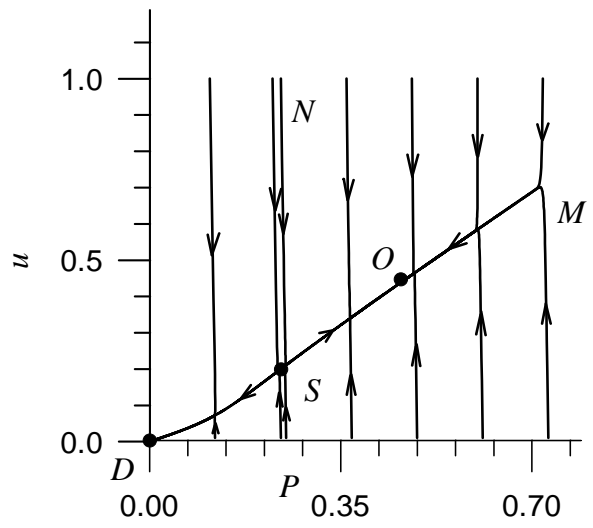

(c)

$v$

Fig. 2. Phase portraits in the $v-u$ plane at $m=1, u_{0}=0.1, S_{0}=1.25 S_{c}$ : (a) $\varepsilon=10^{-2}$; (b) $\varepsilon=1$; (c) $\varepsilon=10^{2}$.

the $\delta$-correlated stochastic function) [26]. Then, within the adiabatic approximation, Eqs. (11) and (12) are reduced to the time-dependencies

$$
\begin{aligned}
& v(t)=\bar{v}+\tilde{v} \xi(t), \quad S(t)=\bar{S}+\tilde{S} \xi(t), \\
& \bar{v} \equiv S_{0} u d(u), \quad \tilde{v} \equiv \sqrt{I_{v}+I_{S} u^{2}} d(u), \\
& \bar{S} \equiv S_{0} d(u), \quad \tilde{S} \equiv \sqrt{I_{S}+I_{v} u^{2}} d(u), \quad d(u) \equiv\left(1+u^{2}\right)^{-1} .
\end{aligned}
$$

Here, deterministic components are reduced to Eqs. (13), whereas fluctuational ones follow from the known property of additivity of variance of independent Gaussian random quantities [26]. Thus, using the slaving principle inherent in synergetics [27] transforms noises of both vertical velocity component $v$ and slope $S$, which are adiabatic initially, to multiplicative form. As a result, a combination of Eqs. (10), (23) and (24) 
leads to the Langevin equation

$$
\dot{u}=f(u)+\sqrt{I(u)} \xi(t), \quad f \equiv-\frac{\partial E}{\partial u},
$$

where the force $f$ is related to the energy $E$ determined by Eq. (15) and an expression for the effective noise intensity

$$
I(u) \equiv I_{u}+\left(I_{v}+I_{S} u^{2}\right) d^{2}(u)
$$

is obtained in accordance with above mentioned property of noise variance additivity. In order to avoid mistakes, one should notice that a direct insertion of Eqs. (23), (24) into (10) results in the appearance of a stochastic addition

$$
\left[I_{u}^{1 / 2}+\left(I_{v}^{1 / 2}+I_{S}^{1 / 2} u\right) d(u)\right] \xi(t),
$$

whose squared amplitude is quite different from the effective noise intensity (26). Moreover, in contrast to expressions (24), a direct use of the adiabatic approximation in Eqs. (11) and (12) reduces the fluctuational additions in Eqs. (23) to the forms: $\tilde{v} \equiv$ $\left(I_{v}^{1 / 2}+I_{S}^{1 / 2} u\right) d(u), \tilde{S} \equiv\left(I_{S}^{1 / 2}-I_{v}^{1 / 2} u\right) d(u)$. The latter is obviously erroneous since the effective noise of the slope $\tilde{S}$ disappears entirely for the horizontal velocity $u=\sqrt{I_{S} / I_{v}}$. The reason for such a contradiction is that the Langevin equation does not permit the usage of usual analysis methods (see Ref. [26]).

To continue in the usual way, let us write the Fokker-Planck equation related to Langevin Eq. (25):

$$
\frac{\partial P(u, t)}{\partial t}=\frac{\partial}{\partial u}\left\{-f(u) P(u, t)+\frac{\partial}{\partial u}[I(u) P(u, t)]\right\} .
$$

At steady state, that will be the only case we consider, the probability distribution $P(u, t)$ becomes a time-independent function $P(u)$ and under the usual condition, that the expression in braces of the right-hand side of Eq. (28) is equal to zero, this leads to a stationary distribution

$$
P(u)=Z^{-1} \exp \{-U(u)\},
$$

where $Z$ is a normalization constant. The effective energy

$$
U(u)=\ln I(u)-\int_{0}^{u} \frac{f\left(u^{\prime}\right)}{I\left(u^{\prime}\right)} \mathrm{d} u^{\prime}, \quad f \equiv-\frac{\partial E}{\partial u},
$$

is determined by the bare energy $E$, Eq. (15) and the noise intensity $I(u)$, Eq. (26) [28]. Combining these expressions, we can find the explicit form of $U(u)$, which is too cumbersome to be reproduced here. The equation which defines the locations of the maxima of the distribution function $P(u)$

$$
x^{3}-S_{0} x^{2}-2 I_{S} x+4\left(I_{S}-I_{v}\right)=0, \quad x \equiv 1+u^{2},
$$

is much simpler. According to Eq. (31), maxima are insensitive to changes in the intensity of the noise $I_{u}$ of the velocity component $u$, but they are determined by the value $S_{0}$ of the sand pile slope and the intensities $I_{v}, I_{S}$ of the noises of the vertical velocity component $v$ and the slope $S$, which acquire the multiplicative character in 
Eq. (26). Hence, for simplicity $I_{u}$ can be set equal to 0 and Eqs. (15), (30) and (26) give the following expression for the effective energy:

$$
\begin{aligned}
U(u)= & \frac{1}{2}\left[\frac{u^{4}}{2}+\left(2-S_{0}-i\right) u^{2}+(1-i)\left(1-S_{0}-i\right) \ln \left(i+u^{2}\right)\right] \\
& +I_{S} \ln \left[g_{S}^{2}(u)+i g_{v}^{2}(u)\right], \quad i \equiv I_{v} / I_{S} .
\end{aligned}
$$

According to Eq. (31), the effective energy (32) has a minimum at $u=0$ if the driven slope $S_{0}$ does not exceed the critical level

$$
S_{c}=1+2 I_{S}-4 I_{v},
$$

whose value increases at increasing intensity of the noise of the sand pile slope, but decreases with one of the vertical velocities. Here, sand grains do not move. In the simple case $I_{v}=0$, the avalanche creation is related to solutions

$$
u_{ \pm}^{2}=\frac{1}{2}\left[S_{0}-3+\sqrt{\left(3-S_{0}\right)^{2}+4\left(2 S_{0}-3+2 I_{S}\right)}\right]
$$

which are obtained from Eq. (31) after elimination of the root $u^{2}=0$. The magnitude of this solution has its minimum

$$
u_{c}^{2}=\frac{1}{2}\left[\left(S_{0}-3\right)-\sqrt{\left(S_{0}+7\right)\left(S_{0}-1\right)}\right]
$$

on the line defined by expression (33) with $I_{v}=0$. At $S_{0}<\frac{4}{3}$ the roots $\pm u_{c}$ are complex, starting from $S_{0}=\frac{4}{3}$ they become zero and at $S_{0}>\frac{4}{3}$ one has real magnitudes $u_{+}=-u_{-}$. In this way, the tricritical point

$$
S_{0}=4 / 3, \quad I_{S}=1 / 6
$$

addresses to the appearance of roots $u_{ \pm} \neq 0$ of Eq. (31) that means avalanche creation. If condition (33) is satisfied, the root $u=0$ corresponds to the minimum of the effective energy (32) at $S_{0}<\frac{4}{3}$, whereas at $S_{0}>\frac{4}{3}$ this root corresponds to the maximum, and the roots $u_{ \pm}$- to symmetrical minima.

Now, we find another condition for the stability of the roots $u_{ \pm}$. Setting the discriminant of Eq. (31) equal to zero, we get the equations

$$
I_{S}=0, \quad I_{S}^{2}-I_{S}\left[\frac{27}{2}\left(1-\frac{S_{0}}{3}\right)-\frac{S_{0}^{2}}{8}\right]+\frac{S_{0}^{3}}{2}=0
$$

the second of which gives

$$
2 I_{S}=\left[\frac{27}{2}\left(1-\frac{S_{0}}{3}\right)-\frac{S_{0}^{2}}{8}\right] \pm\left\{\left[\frac{27}{2}\left(1-\frac{S_{0}}{3}\right)-\frac{S_{0}^{2}}{8}\right]^{2}-2 S_{0}^{3}\right\}^{1 / 2} .
$$

This equation defines a bell-shaped curve $S_{0}\left(I_{S}\right)$, which intersects the horizontal axis at the points $I_{S}=0$ and $I_{S}=27 / 2$, and has a maximum $S_{0}=2$ at

$$
I_{S}=2 \text {. }
$$

It is easy to see that for $I_{v}=0$ this line touches the curve (33) at point (36). 

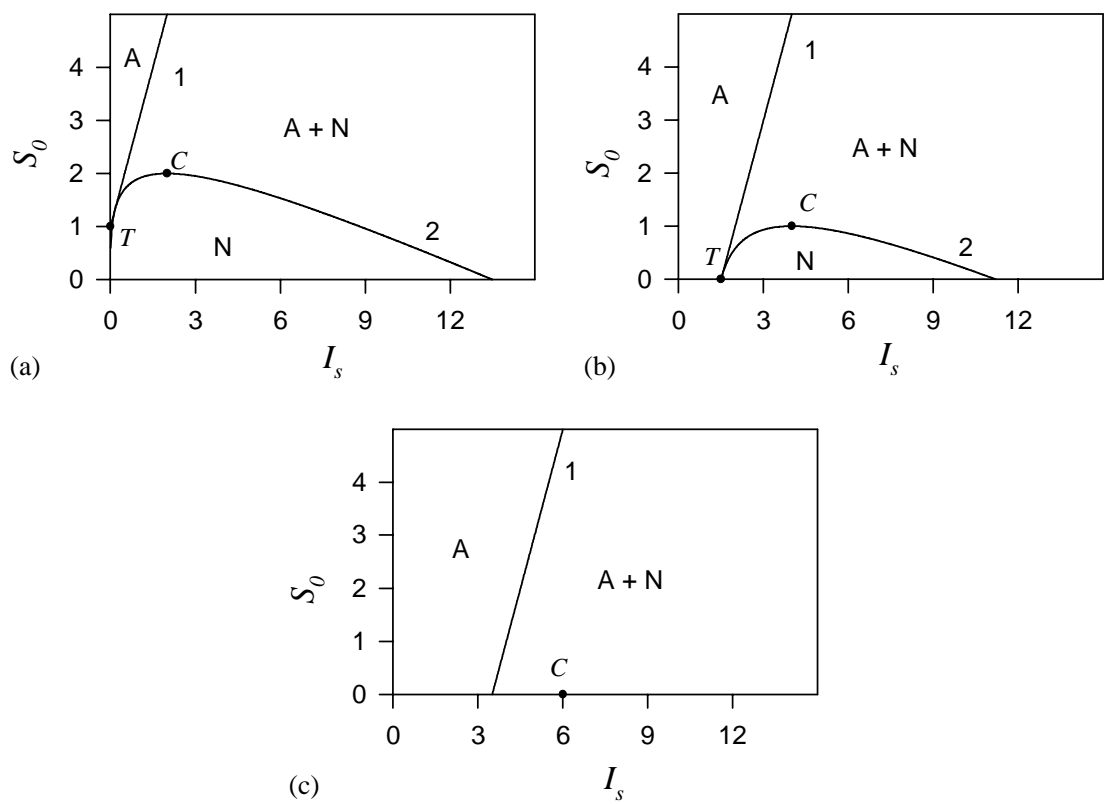

Fig. 3. Phase diagrams at fixed values $I_{v}$ : (a) $I_{v}=0$; (b) $I_{v}=1$; (c) $I_{v}=2$. Curves 1 and 2 define the boundary of stability of avalanche $(A)$ and non-avalanche $(\mathrm{N})$ phases.

Let us now consider the more general case of two multiplicative noises $I_{v}, I_{S} \neq 0$. Introducing the parameter $a=1-i, i \equiv I_{v} / I_{S}$ and the renormalized variables $\tilde{I} \equiv I_{S} / a^{2}$, $\tilde{S}_{0} \equiv S_{0} / a, \tilde{u}^{2}=\left(1+u^{2}\right) / a-1$, at $i<1$ we reproduce all of the above expressions with the generalized energy $\tilde{U} / \tilde{I}$ in Eq. (32). Thus, the action of the noise of the vertical velocity component $v$ is reduced to the renormalization of the extremum value of the horizontal one $u$ by the quantity $\left(a^{-1}-1\right)^{1 / 2}$. As a result, the region of divergence $\tilde{u} \approx 0$ becomes inaccessible.

The condition of extremum of the generalized energy (32) splits into two equations, one of which is simply $u=0$, and the other one is given by Eq. (31). As mentioned above, the analysis of the latter indicates that the line of existence of the zero solution is defined by expression (33). The tricritical point has the coordinates

$$
S_{0}=\frac{4}{3}\left(1-I_{v}\right), \quad I_{S}=\frac{1}{6}\left(1+8 I_{v}\right) .
$$

The phase diagram for the fixed intensities $I_{v}$ is shown in Fig. 3. Here the curves 1, 2 define the thresholds of absolute loss of stability for the fluxless and flux steady-states, respectively. Above line 1 the system is in a stable flux state, below curve 2 it is in the fluxless one, and between these lines the two-phase domain is realized. For $I_{v}<\frac{1}{4}$ the situation is generally the same as in the simple case $I_{v}=0$ (see Fig. 3a). At $I_{v}>\frac{1}{4}$ even for small intensities $I_{S}$ of the slope noise (Fig. 3b) the avalanche formation is possible. According to Eq. (40), the tricritical point lies on the $I_{S}$-axis at $I_{v}=1$, and if the noise intensity $I_{v}$ is larger than the critical value $I_{v}=2$, the stable fluxless state disappears (see Fig. 3c). 
To conclude, the above consideration shows that the dissipative dynamic of grain flow in a real sand pile can be represented within the framework of the Lorenz model, where the horizontal and vertical velocity components play the role of the order parameter and its conjugate field, respectively, and the sand pile slope is the control parameter. In Section 2, the noiseless case is examined to show that an avalanche is created if the externally driven sand pile slope $y_{0}^{\prime}$ is larger than the critical magnitude

$$
y_{c}^{\prime}=\left(\tau_{x} \tau_{y}\right)^{-1 / 2} \gamma .
$$

In this sense, the systems with small values of the kinetic coefficient $\gamma$ and large relaxation times $\tau_{x}, \tau_{y}$ of the velocity components are preferred. However, the sand flow appears as usual phase transition because the avalanche creation in the noiseless case is only possible due to the externally driven growth of the sand pile slope.

A consideration of the additive noises of the above degrees of freedom shows that the stochasticity influence is non-essential for the horizontal velocity component and that it is crucial for both the vertical velocity component and the sand pile slope. The boundary of the domain of avalanche formation is set by the equality for the dimensionless noise intensities

$$
I_{S}=-\frac{1}{2}+2 I_{v}
$$

following from Eq. (31) at the conditions $x=1(u=0)$, and $S_{0}=0$. According to Eq. (42), in absence of the sand pile slope noise the avalanche is created if the intensity of the vertical velocity component exceeds the value

$$
I_{v 0}=\frac{1}{4} \frac{D \gamma^{2}}{\tau_{x} \tau_{y} \tau_{S}}
$$

corresponding to the point $O$ in Fig. 4. An increase of both the vertical velocity and the sand pile slope noises causes an avalanche formation if its intensities are bound by condition (42). The domain of the mixed state appears with further increase of these intensities above magnitudes

$$
I_{v 1}=\frac{D \gamma^{2}}{\tau_{x} \tau_{y} \tau_{S}}, \quad I_{S 1}=\frac{3}{2} \frac{\gamma^{2}}{\tau_{x} \tau_{y}}
$$

at the point $T$ in Fig. 4. If the noise intensity of the vertical velocity exceeds the larger value

$$
I_{v 2}=2 \frac{D \gamma^{2}}{\tau_{x} \tau_{y} \tau_{S}},
$$

corresponding to the sand pile slope noise $I_{S 2}=6 \gamma^{2} / \tau_{x} \tau_{y}$ (the point $C$ in Fig. 4 ), the fluxless steady-state disappears at all.

Physically, we have to take into consideration that the SOC regime is not relevant to a flux-type avalanche state itself, but rather to an intermittent regime of avalanche formation corresponding to the domains in the phase diagrams in Figs. 3 and 4, where a mixture of both phases $\mathrm{A}$ and $\mathrm{N}$ (avalanche and non-avalanche) exists. According to the above analysis, such an intermittent behavior may be realized within the region 


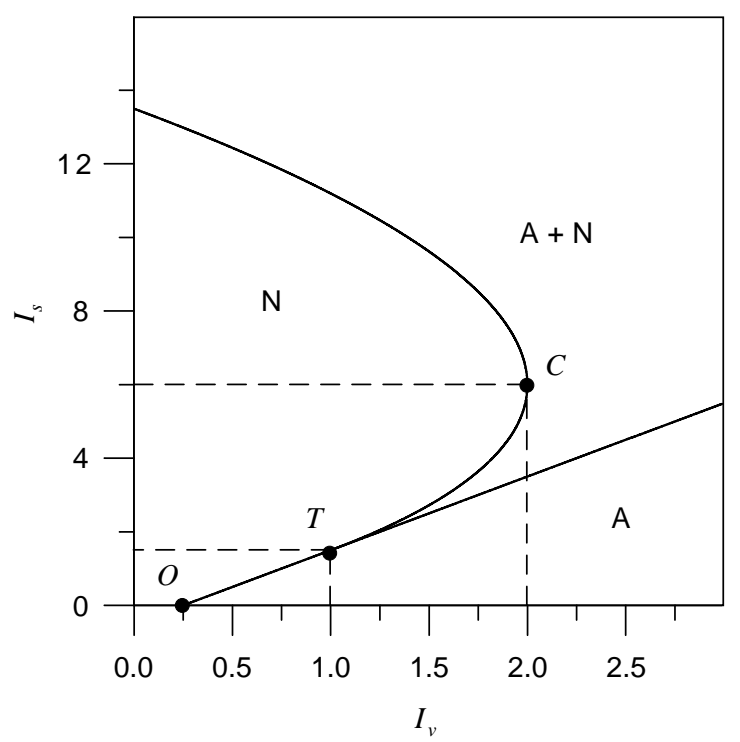

Fig. 4. Phase diagram for the system with $S_{0}=0$ and $I_{s}, I_{v} \neq 0$ ( $D$ is the disordering point; $T$ is the tricritical point; $C$ is the critical point).

located above line (42) and outside the curve that is determined by

$$
I_{v}=I_{S}\left[1-\left(\frac{2}{27}\right)^{1 / 2} \sqrt{I_{S}}\right]
$$

with the dimensionless values $I_{v}, I_{S}$. The corresponding phase diagram is depicted in Fig. 4 to show a very non-trivial form (especially, within the domain $I_{v 1} \leqslant I_{v} \leqslant I_{v 2}$ ).

\section{Generalizing self-similarity}

To proceed the consideration of the system behavior, let us examine the explicit form of the probability (29) determined, for different regimes, by the effective energy (30). In the case $I_{u}, I_{S} \ll I_{v}$, we obtain a distribution

$$
\begin{aligned}
& P(u) \approx I_{v}^{-1}\left(1+u^{2}\right)^{2} \exp \left\{I_{v}^{-1} \int f(u)\left(1+u^{2}\right)^{2} \mathrm{~d} u\right\}, \\
& f(u) \equiv-u+S_{0} u /\left(1+u^{2}\right)
\end{aligned}
$$

that differs from the power dependence inherent in self-similar systems. Contrary, at intermittent behavior, when $I_{u}, I_{v} \ll I_{S}$, the supercritical values of the slope noise intensity $I_{S}$ cause the following distribution form

$$
P(u) \approx I_{S}^{-1}\left(\frac{1+u^{2}}{u}\right)^{2} \exp \left\{I_{S}^{-1} \int \frac{f(u)\left(1+u^{2}\right)^{2}}{u^{2}} \mathrm{~d} u\right\} \sim u^{-2} .
$$


Thus, the case $I_{u}, I_{v} \ll I_{S}$ addresses to the power-law distribution that is relevant to self-similar behavior. However, in general case, the obtained exponent should not be reduced to the integer 2 but to fractional one.

To get rid off such a restriction, the multiplier $u$ in the nonlinear terms of Eqs. (10)-(12) is supposed to be replaced by power term $u^{a}$, with an exponent $0 \leqslant a \leqslant 1$. Taking into account the stochastic additions, one obtains the basic equations in dimensionless form

$$
\begin{aligned}
& \dot{u}=-u+v+\sqrt{I_{u}} \xi(t), \\
& \varepsilon \dot{v}=-v+u^{a} S+\sqrt{I_{v}} \xi(t), \\
& \delta \dot{S}=\left(S_{0}-S\right)-u^{a} v+\sqrt{I_{S}} \xi(t) .
\end{aligned}
$$

It can be seen that the agreement of the Lorenz self-organization scheme with SOC conception, related to self-similar systems is achieved, if one assumes that both positive and negative feedbacks are fractional. Within such a supposition, the adiabatic approximation $\varepsilon, \delta \ll 1$ leads to the Langevin equation (cf. Eq. (25))

$$
\dot{u}=f_{a}(u)+\sqrt{I_{a}(u)} \xi(t),
$$

where the force $f_{a}(u)$ and the noise intensity $I_{a}(u)$ are as follows:

$$
\begin{aligned}
& f_{a}(u) \equiv-u+S_{0} u^{a} d_{a}(u), \\
& I_{a}(u) \equiv I_{u}+\left(I_{v}+I_{S} u^{2 a}\right) d_{a}^{2}(u), \quad d_{a}(u) \equiv\left(1+u^{2 a}\right)^{-1} .
\end{aligned}
$$

The corresponding distribution (cf. Eqs. (29) and (30))

$$
P_{a}(u)=\frac{Z^{-1}}{I_{a}(u)} \exp \left\{-E_{a}(u)\right\},
$$

where $Z$ is the partition function, is determined by an effective potential

$$
E_{a}(u) \equiv-\int_{0}^{u} \frac{f_{a}\left(u^{\prime}\right)}{I_{a}\left(u^{\prime}\right)} \mathrm{d} u^{\prime}
$$

The extremum points of this distribution are determined by the equation

$$
2 a I_{S} u^{2 a}+\left(1+u^{2 a}\right)^{2} u^{1-a}\left[S_{0}-u^{1-a}\left(1+u^{2 a}\right)\right]=2 a\left(I_{S}-2 I_{v}\right),
$$

according to which the boundary of the flux state

$$
I_{S}=2 I_{v}
$$

relates to the condition $u=0$. Critical values of state parameters are fixed by the condition $\left|\mathrm{d} u / \mathrm{d} S_{0}\right|=\infty$ leading to additional equation

$$
\begin{aligned}
& u^{2(1-a)}\left(1+u^{2 a}\right)^{2}\left[\left(2+a^{-1}\right)+\left(a^{-1}-1\right) u^{-2 a}\right] \\
& \quad-\frac{1}{2} S_{0} u^{1-a}\left(1+u^{2 a}\right)\left[\left(3+a^{-1}\right)+\left(a^{-1}-1\right) u^{-2 a}\right]=2 a I_{S} .
\end{aligned}
$$

Expressions (54)-(56) generalize the simple equalities (31), (42) and (46) related to the case $a=1$. 


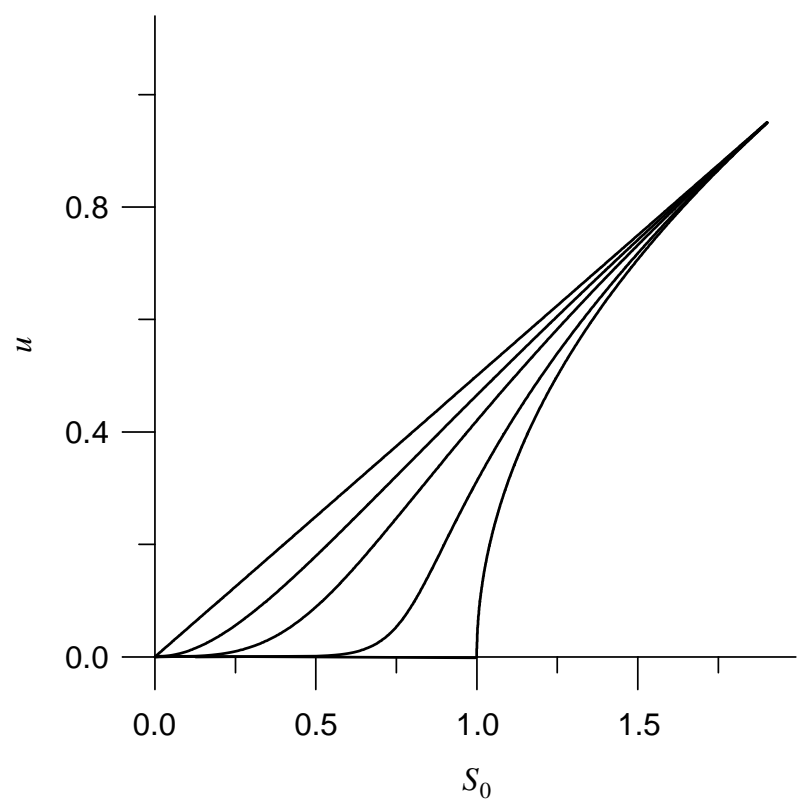

Fig. 5. The $S_{0}$-dependence of the steady-state velocity $u$ at $a=0,0.5,0.7,0.9,1.0$ from top to bottom.

Above expressions show that qualitative results of Section 3 obtained for the particular case $a=1$ are kept valid with the passage to the general case $0 \leqslant a \leqslant 1$. Indeed, the most essential difference is observed for the noiseless case, namely the steady-state velocity $u$ becomes non-zero within the whole interval of the driven slope $S_{0}$ (see Fig. 5). An increase of the vertical velocity noise $I_{v}$ causes monotonic $u$-growth, whereas $I_{S}$-increase leads to an effective barrier formation near the point $u=0$, so that the dependence $u\left(S_{0}\right)$ becomes non-monotonic at magnitudes $I_{S}$ above the straight line (55) (see Fig. 6). Here, by analogy with noiseless case (see Fig. 1), lower branches of curves correspond to unstable magnitudes of the order parameter, while the upper meet the stable ones. According to Fig. 7, the domain, where avalanches can not be created, is located near intermediate magnitudes of the state parameters $S_{0}, I_{v}, I_{S}$. The phase diagram related to the avalanche formation reveals the same form as for the simplest case $a=1$, but the straight line (42) shifts abruptly to (55) with escaping the point $a=1$ (compare Fig. 8 with Fig. 4). According to Fig. 9, an increase of the vertical velocity noise $I_{v}$ increases the domain of the avalanche formation.

\section{Size distribution in self-similar ensemble of avalanches}

In contrast to the previous discussion, where the process of a single avalanche formation has been considered, now we will study analytically the self-similar size distribution (2) of an avalanche ensemble. This means that, along the line of Section 3, 

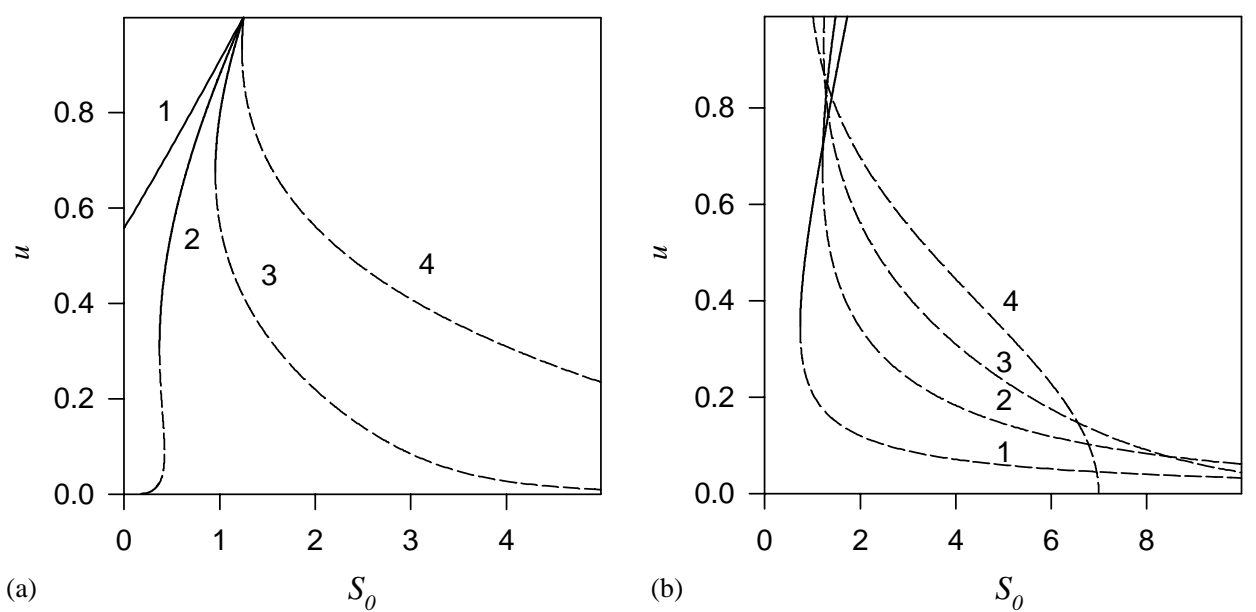

Fig. 6. The $S_{0}$-dependence of the steady-state velocity $u$ : (a) at $a=0.75, I_{v}=1$ (curves $1-4$ are related to $\left.I_{S}=1,2,3,5\right) ;(\mathrm{b})$ at $I_{v}=1, I_{S}=5$ (curves $1-4$ are related to $a=0.25,0.5,0.75,1.0$ ).

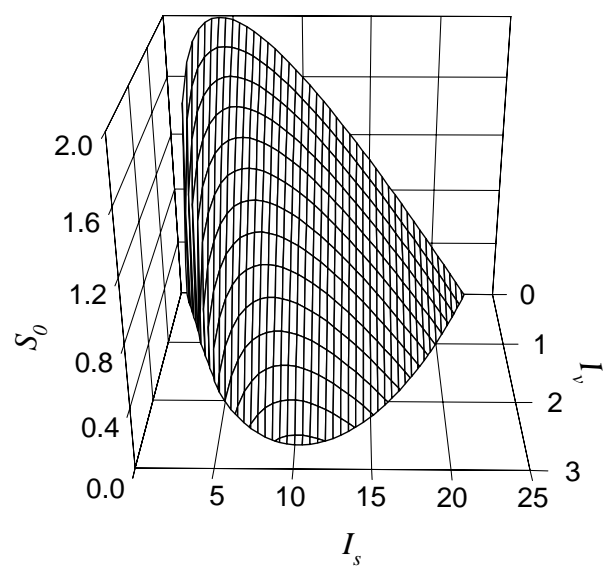

Fig. 7. Three-dimensional phase diagram (the non-avalanche domain is located under the surface).

we will account for noises of a complete set of degrees of freedom, on the one hand, and the fractional feedback type introduced in Section 4, on the other one. Thereby, the Lorenz system unified in the above manner is the basis of our examination. However, instead of visible geometric-and-mechanic characteristics of a 'real' sand pile, the system under consideration is now parameterized by a set of pseudo-thermodynamical variables, that describes the avalanche ensemble in the spirit of the famous Edwards paradigm [22,23] generalized to non-stationary system. With this method, we study time dependencies of the avalanche size, non-extensive complexity and non-conserved energy of the moving grains. Within the framework of the usual synergetic approach, 


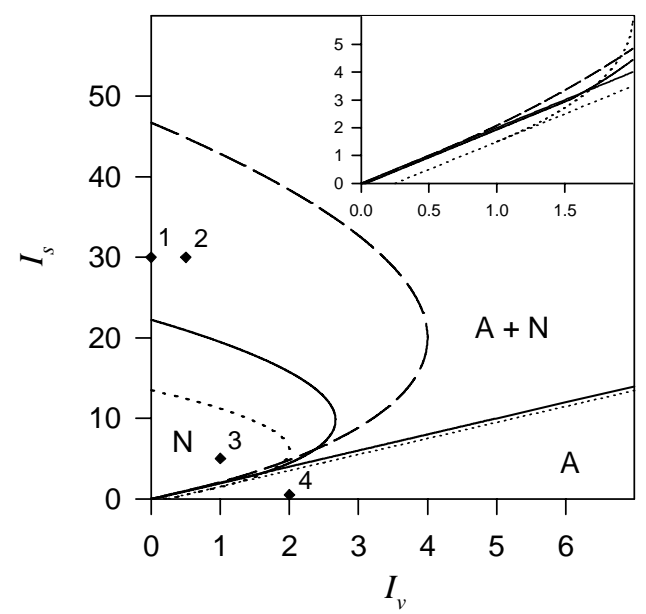

Fig. 8. Phase diagram for the system with $S_{0}=0$ and $I_{s}, I_{v} \neq 0$ at $a=0.5,0.75,1.0$ (dashed, solid and dotted curves, respectively). Diamonds are related to curves $1-4$ in Fig. 11.

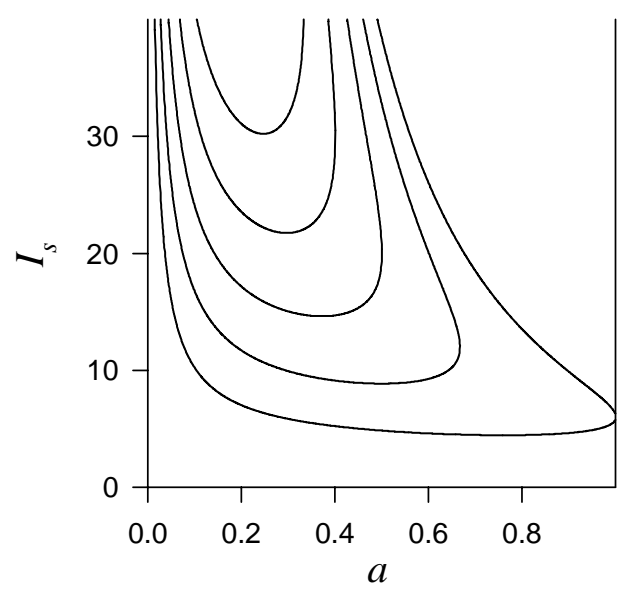

Fig. 9. Phase diagram in the $I_{S}-a$ plane at $I_{v}=2,3,4,5,6$ from bottom to top (the non-avalanche domain is located inside the curves).

these degrees of freedom play the role of order parameter, conjugate field and control parameter, respectively.

It is principally important that the use of the slaving principle of both synergetics and fractional nature of the system feedback is shown to stipulate the multiplicative character of noise. It will be shown, that this causes a non-extensivity of the applied thermodynamical scheme, so that we have to use $q$-weighted averages instead of usual 
ones. So, energy of moving sand grains is defined by the expression

$$
\zeta_{q} \equiv \sum_{i} \zeta_{i} p_{i}^{q}
$$

where $p_{i}$ is a probability to move grain $i$ with energy $\zeta_{i}, q \neq 1$ is a positive parameter that is a measure for the system's non-extensivity determined below. Non-extensive complexity of moving sand grains is an analog to Tsallis entropy [24] that is determined as follows:

$$
\Sigma_{q} \equiv-\frac{\sum_{i} p_{i}^{q}-1}{q-1} .
$$

The three-parameter set of the standard synergetic scheme [27] is completed by the avalanche size $s$.

Following the above elaborated line, we postulate that a self-consistent behavior of the considered system is presented adequately by a set of pointed out quantities governed by the Lorenz-type equations (cf. Eqs. (49))

$$
\begin{aligned}
& \tau_{s} \dot{s}=-s+a_{s} \Sigma_{q}+\sqrt{I_{s}} \xi(t), \\
& \tau_{\Sigma} \dot{\Sigma}_{q}=-\Sigma_{q}+a_{\Sigma} s^{\tau / 2} \zeta_{q}+\sqrt{I_{\Sigma}} \xi(t), \\
& \tau_{\zeta} \dot{\zeta_{q}}=\left(\zeta^{0}-\zeta_{q}\right)-a_{\zeta} s^{\tau / 2} \Sigma_{q}+\sqrt{I_{\zeta}} \xi(t) .
\end{aligned}
$$

Here $\tau_{s}, \tau_{\Sigma}, \tau_{\zeta}$ note relaxation times of corresponding values, $a_{s}, a_{\Sigma}, a_{\zeta}$ are related feedback parameters, $I_{s}, I_{\Sigma}, I_{\zeta}$ are respective noise intensities, $\tau$ is a positive exponent and $\zeta^{0}$ is the externally driven energy of the sand motion. The distinguishing feature of the first of these equations is that in a noiseless case genuine characteristics $s, \Sigma_{q}$ are linearly connected. On the other hand, the two last equations (59) show that the connection of values $\zeta_{q}, \Sigma_{q}$, that are of a thermodynamic type, with the avalanche size $s$ is nonlinear. Physically, this means a linear relation between the complexity and the avalanche size near steady state. Moving away this leads to negative feedback of the avalanche size and the complexity on the energy that, in accordance with Le Chatelier principle, results in the energy decrease. Moreover, positive feedback appears of the avalanche size and the energy on the complexity, which causes complexity increase that is the reason for the avalanche ensemble's self-organization.

To analyze system (59), it is convenient to measure the time $t$ in unit $\tau_{s}$ and introduce the scales for variables $s, \Sigma_{q}, \zeta_{q}, I_{s}, I_{\Sigma}$, and $I_{\zeta}$ as follows:

$$
\begin{aligned}
& s^{s c} \equiv\left(a_{\Sigma} a_{\zeta}\right)^{-1 / \tau}, \quad \sum_{q}^{s c} \equiv a_{s}^{-1}\left(a_{\Sigma} a_{\zeta}\right)^{-1 / \tau}, \quad \zeta_{q}^{s c} \equiv a_{s}^{-1} a_{\Sigma}^{-(1 / \tau+1 / 2)} a_{\zeta}^{-(1 / \tau-1 / 2)}, \\
& I_{s}^{s c} \equiv\left(a_{\Sigma} a_{\zeta}\right)^{-2 / \tau}, \quad I_{\Sigma}^{s c} \equiv a_{s}^{-2}\left(a_{\Sigma} a_{\zeta}\right)^{-2 / \tau}, \quad I_{\zeta}^{s c} \equiv a_{s}^{-2} a_{\Sigma}^{-(2 / \tau+1)} a_{\zeta}^{-(2 / \tau-1)} .
\end{aligned}
$$

Then, the scaled Lorenz system (59) takes the simple form

$$
\begin{aligned}
& \dot{s}=-s+\Sigma_{q}+\sqrt{I_{s}} \xi(t), \\
& \vartheta \dot{\Sigma}_{q}=-\Sigma_{q}+s^{\tau / 2} \zeta_{q}+\sqrt{I_{\Sigma}} \xi(t), \\
& \theta \dot{\zeta_{q}}=\left(\zeta^{0}-\zeta_{q}\right)-s^{\tau / 2} \Sigma_{q}+\sqrt{I_{\zeta}} \xi(t),
\end{aligned}
$$


with the ratios of relaxation times

$$
\vartheta \equiv \tau_{\Sigma} / \tau_{s}, \quad \theta \equiv \tau_{\zeta} / \tau_{s}
$$

It is worth to notice that system (61) is passed to the form of Eqs. (49) if the values $s, \Sigma_{q}, \zeta_{q}, \tau / 2, \vartheta$, and $\theta$ are replaced by $u, v, S, a, \varepsilon$, and $\delta$, respectively.

It is well-known that a complete set of SOC systems can be reduced to one of two families [19]: systems with deterministic dynamics extremely driven by a random environment (growing interface models, Bak-Sneppen evolution model etc.) and the stochastic dynamics family (models of earthquakes, forest-fire etc.). ${ }^{2}$ A remarkable peculiarity of the obtained system (61) is the possibility to present both mentioned families in a natural manner. The former is related to the noiseless case, when $I_{S}$, $I_{\Sigma}, I_{\zeta}=0$ but the magnitude of the energy relaxation time is larger than that of the complexity and avalanche size $\left(\tau_{\zeta} \geqslant \tau_{\Sigma}, \tau_{s}\right)$; on the other hand, a parameter of the environment drive $\zeta^{0}$ has to take a larger value than the critical one $\zeta_{c}=1$ [17]. In such a case, system (61) describes a strange attractor that may represent the behavior of SOC systems of the first type. A proper stochastic behavior is relevant for non-vanishing to non-zeroth noise intensities $I_{s}, I_{\Sigma}, I_{\zeta} \neq 0$ that make possible the appearance of the SOC regime even in the absence of a driven affect $\left(\zeta^{0}=0\right)$.

Taking into account that the problem of the Lorenz strange attractor is well-known [27], we will restrict ourselves to the treatment of the stochastic system, where the adiabatic conditions $\vartheta, \theta \ll 1$ are applicable. Then, the two last equations of system (61) lead to dependencies of the type of Eqs. (23)

$$
\Sigma_{q}(t)=\bar{\Sigma}_{q}+\tilde{\Sigma}_{q} \xi(t), \quad \zeta_{q}(t)=\bar{\zeta}_{q}+\tilde{\zeta}_{q} \xi(t),
$$

where the deterministic and the fluctuational components are determined as follows (cf. Eqs. (24))

$$
\begin{aligned}
& \bar{\Sigma}_{q} \equiv \zeta^{0} s^{\tau / 2} d_{\tau}(s), \quad \tilde{\Sigma}_{q} \equiv \sqrt{I_{\Sigma}+I_{\zeta} s^{\tau}} d_{\tau}(s), \\
& \bar{\zeta}_{q} \equiv \zeta^{0} d_{\tau}(s), \quad \tilde{\zeta}_{q} \equiv \sqrt{I_{\zeta}+I_{\Sigma} s^{\tau}} d_{\tau}(s), \quad d_{\tau}(s) \equiv\left(1+s^{\tau}\right)^{-1} .
\end{aligned}
$$

Due to the slaving principle of synergetics, the initially adiabatic noises of the complexity and the energy are transformed to a multiplicative form. On the other hand, the relation between the complexity and energy

$$
\bar{\Sigma}_{q}=\sqrt{\bar{\zeta}_{q}\left(\zeta^{0}-\bar{\zeta}_{q}\right)}
$$

that can be deduced with the dependencies (64), leads to the expression

$$
T=-\left(1-\frac{\zeta^{0}}{2 \bar{\zeta}_{q}}\right)^{-1} \sqrt{\frac{\zeta^{0}}{\bar{\zeta}_{q}}-1}
$$

for the effective temperature $T \equiv \partial \bar{\zeta}_{q} / \partial \bar{\Sigma}_{q}$. As depicted in Fig. 10a, $T$ is a monotonically increasing function of the energy with boundary values $T\left(\bar{\zeta}_{q}=0\right)=0$ and $T\left(\bar{\zeta}_{q}=\zeta^{0} / 2\right)=$ $\infty$. At the latter point the magnitude $T$ changes instantaneously to $-\infty$ and then increases monotonically again to initial value $T=0$ at $\bar{\zeta}_{q}=\zeta^{0}$. This means that in the

\footnotetext{
${ }^{2}$ In general, we deal with a much more complicated problem, see Ref. [29].
} 


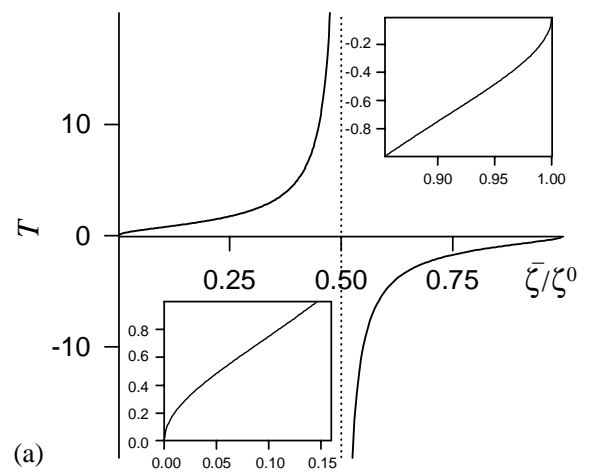

Fig. 10. The energy dependences of the avalanche ensemble temperatures: (a) nonstationary magnitude $T$ versus ratio $\bar{\zeta}_{q} / \zeta^{0} ;$ (b) stationary temperature $T_{0}$ versus $\zeta^{0}$.

domain $0 \leqslant \bar{\zeta}_{q}<\zeta^{0} / 2$ the avalanche system is dissipative and behaves in usual manner; on the contrast, in the domain $\zeta^{0} / 2<\bar{\zeta}_{q} \leqslant \zeta^{0}$ a self-organization process evolves, so that an energy increase leads to a complexity decrease, in accordance with a negative temperature. At steady state, where an avalanche has got a stationary size $s_{0}=\sqrt{\zeta^{0}-1}$, the temperature takes the stationary value

$$
T_{0}=-\frac{\sqrt{\zeta^{0}-1}}{1-\zeta^{0} / 2}
$$

that is negative in the supercritical domain $1 \leqslant \zeta^{0}<2$. According to Fig. 10b, the magnitude $T_{0}$ decreases monotonically with the driven energy from value $T_{0}\left(\zeta^{0}=1\right)=0$ to $T_{0}\left(\zeta^{0} \rightarrow 2\right) \rightarrow-\infty$.

The presented self-organization regime relates to externally driven systems, which are relevant for the usual phase transition but not to the SOC itself. To study the latter within the above consideration, let us combine Eqs. (63) and (64) with the first of the Eqs. (61) in that way that has been used above for obtaining the Langevin equation (25). By analogy with Section 4, this leads to stochastic equation (50), where the effective force and noise intensity are given by Eqs. (51) with accuracy to the replacements mentioned after Eqs. (62): the quantities $s, \Sigma_{q}, \zeta_{q}, \tau / 2$ have to be taken instead of $u, v, S, a$, respectively. Then, all results obtained in Section 4 can be used immediately. Particularly, it is found that the influence of a random scattering of the avalanche size is non-essential, whereas energy and complexity noises lead to a crucial effect. The related picture is reflected by Fig. 8 taken in plane $I_{\zeta}-I_{\Sigma}$ that is formed by corresponding noise intensities of the avalanche ensemble. The mixed domain $\mathrm{A}+\mathrm{N}$ with respect to the intermittency regime is bounded by the straight line (55) and the bell-shaped curve type of Eqs. (46). According to Fig. 9, where exponent $a$ has to be replaced by $\tau / 2$, the random scattering growth of the complexity extends the SOC domain along the axis of the exponent $\tau$. 


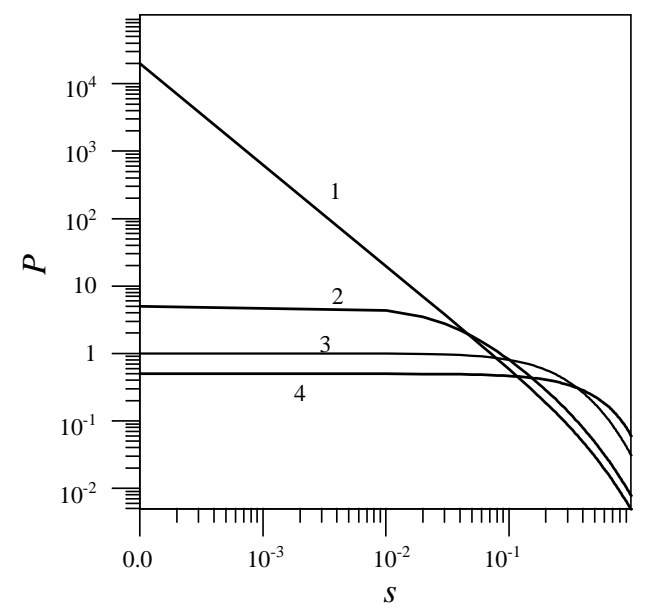

Fig. 11. Distribution function (68) at $\tau=1.5$ and regimes pointed out by diamonds in Fig. 8: (1) $I_{v}=0$, $I_{S}=30(\mathrm{SOC}) ;(2) I_{v}=0.5, I_{S}=30(\mathrm{~A}+\mathrm{N}) ;(3) I_{v}=1, I_{S}=5(\mathrm{~N}) ;(4) I_{v}=2, I_{S}=0.5(\mathrm{~A})$.

Now, we will discuss the distribution of the avalanche size on basis of Eqs. (51)-(53). For arbitrary noise intensities one has

$$
\begin{aligned}
& P(s)=\frac{Z^{-1}}{I(s)} \exp \left\{\int_{0}^{s} \frac{f\left(s^{\prime}\right)}{I\left(s^{\prime}\right)} \mathrm{d} s^{\prime}\right\}, \\
& f(s) \equiv-s+\zeta^{0} s^{\tau / 2} d_{\tau}(s), \\
& I(s) \equiv I_{s}+\left(I_{\Sigma}+I_{\zeta} s^{\tau}\right) d_{\tau}^{2}(s), \quad d_{\tau}(s) \equiv\left(1+s^{\tau}\right)^{-1} .
\end{aligned}
$$

In the SOC regime the driven energy is vanished, $\zeta^{0}=0$ and the distribution (68) behaves as depicted in Fig. 11 for different noise intensities of both energy and complexity. It can be seen that the power-law dependence inherent in the SOC regime is observed only in the limits $s \ll 1$ and $I_{s}, I_{\Sigma} \ll I_{\zeta}$. In this case, the distribution (68) is reduced to the canonical form (2), where the second multiplier takes the form

$$
\mathscr{P}(s)=\frac{d_{\tau}^{-2}(s)}{Z} \exp \left\{-I_{\zeta}^{-1} \int_{0}^{s} \frac{d_{\tau}^{-2}\left(s^{\prime}\right)}{\left(s^{\prime}\right)^{\tau-1}} \mathrm{~d} s^{\prime}\right\}, \quad d_{\tau}(s) \equiv\left(1+s^{\tau}\right)^{-1} .
$$

It is easy to see that the deviation of this multiplier from a constant value is estimated with term $\sim s^{2-\tau}$, that increases with decrease of $\tau$ and growth of avalanche size to extremely large magnitudes $s \sim 1$, i.e., with escaping SOC domain. This is confirmed by Fig. 12, where the deviation $\delta \tau$ of the slope of dependence $P(s)$, Eqs. (68) in the linear domain from the theory parameter $\tau$ is depicted as a function of the parameter $\tau$ itself. In accordance with the above estimation, it can be seen that the deviation $\delta \tau$ takes a maximal value $\delta \tau<10^{-1} \tau$ at non-essential magnitudes $\tau<1$ or, with noise intensity growth to enormous values $I_{S} \sim 10^{3}$. 


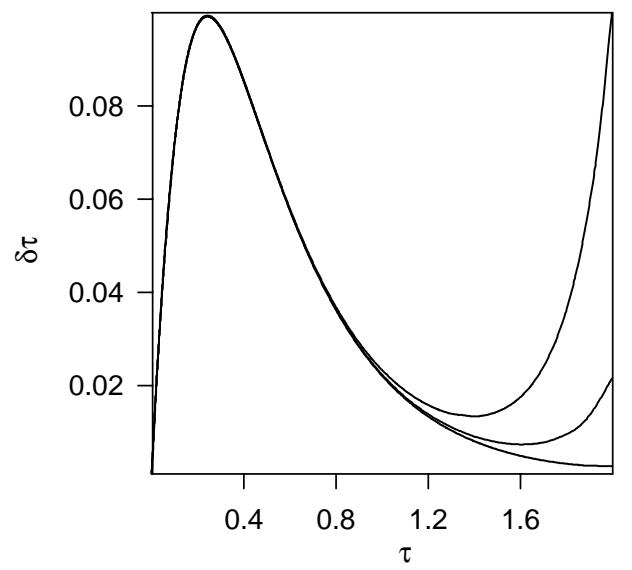

Fig. 12. Deviation $\delta \tau$ of the linear slope of curve 1 depicted in Fig. 11 from parameter $\tau$ versus the exponent $\tau$ itself $\left(I_{S}=10,50,10^{3}\right.$ from bottom to top $)$.

\section{Discussion}

A remarkable peculiarity of expression (69) is that, within the limits $s \ll 1, I_{s}, I_{\Sigma} \ll I_{\zeta}$ inherent in the SOC regime, it can be expressed in terms of standard Gamma-function $\Gamma(x)$ and fractional integral $\mathscr{I}_{-s}^{2-\tau}$ of order $2-\tau$ (see Appendix A and, for details, Refs. [30,31])

$$
\mathscr{P}(s)=\frac{d_{\tau}^{-2}(s)}{Z} \exp \left\{-\frac{\Gamma(2-\tau)}{I_{\zeta}} \mathscr{I}_{-s}^{2-\tau} d_{\tau}^{-2}(s)\right\} .
$$

On the other hand, it is well-known [25] that expressions of this kind appear as a solution of the fractional Fokker-Planck equation

$$
\mathscr{D}_{t}^{\omega} \mathscr{P}(s, t)=\mathscr{D}_{-s}^{\varpi}\left\{s \mathscr{P}(s, t)+\frac{I_{\zeta}}{\Gamma(\varpi)} \mathscr{D}_{-s}^{\varpi}\left[d_{\tau}^{2} \mathscr{P}(s, t)\right]\right\},
$$

where the fractional derivative $\mathscr{D}_{x}^{\varpi}$ (see (A.2)) is used to be inverted to the fractional integral (A.1). Multiplying Eq. (71) by term $s^{2 \pi}$ and averaging over $s$ according to the definitions,

$$
|s| \equiv\left\langle s^{\alpha}\right\rangle^{1 / \alpha}, \quad\left\langle s^{\alpha}\right\rangle \equiv \int_{-\infty}^{\infty} s^{\alpha} \mathscr{P}(s, t) \mathrm{d} s, \quad \alpha>0,
$$

one obtains at $\alpha \equiv 2 \varpi$

$$
|s|^{z} \sim t, \quad z=\frac{2 \varpi}{\omega},
$$

where $z$ is a dynamical exponent. This relation corresponds to the large time limit, where only the diffusional contribution is essential. Combining expressions (70), (73) and (A.1) leads to the relations $2-\tau=\varpi=z \omega / 2$, that yield

$$
\tau=2-\frac{z \omega}{2} \text {. }
$$


Comparing this equation with the second of the known relations (1), one obtains

$$
\omega z=\frac{4}{D} \text {. }
$$

The mean-field magnitudes $\omega=1$ and $D=4$ are related to the dynamical exponent $z=1$ that, in accordance with definition (73), is related to the unusual ballistic limit of the SOC regime. On the other hand, the fractional Fokker-Planck equation (71) leads to the usual diffusional regime with $z=2$ only in the artificial case, when the time-derivative exponent is assumed to be $\omega=\frac{1}{2}$.

The obvious reason for a such discrepancy is the non-consistent application of the usual field relations (1) to the Lorenz system (61). In this system, the stochastic degrees of freedom $s, \Sigma_{q}$ and $\zeta_{q}$, whose number is $n=3$, serve as the different space directions. However, the stochastic process evolves for any of these variables in a plane spanned by the given variable itself and its conjugated momentum. Moreover, the multiplicative character of noise, which is determined by the exponent $a$ in expressions (51), reduces the fractal dimension of every plane to the value $2(1-a)$ [28]. Thus, the resulting fractal dimension of the phase space, in that the stochastic system evolves, is as follows:

$$
D=2 n(1-a),
$$

where $n=3$ for the used Lorenz system. Inserting this dimension into expression (75) leads to $\omega z=2$, which, in contrast to the relation $\omega z=1$ obtained above, is correct in the simplest case $\omega=1, z=2$ [the latter is relevant to a single stochastic degree of freedom $(n=1)$ with additive noise $(a=0)$ ]. In the general case, Eqs. (74)-(76) yield the final result

$$
\tau=2\left[1-\frac{1}{2 n(1-a)}\right] .
$$

The respective dependencies are depicted in Figs. 13a,b to show that the exponent $\tau$ increases monotonically from its minimum magnitude $\tau=1$ at the critical number $(1-a)^{-1}$ to upper value $\tau=2$ in the limit $n \rightarrow \infty$; thereby, an $a$-growth shifts the dependence $\tau(n)$ to large magnitudes $n$, i. e., decreases the exponent $\tau$.

It is easy to see that relation (77) reproduces known results of different approaches for the dimension $D$ (see Ref. [32]). In the case related to mean-field theory, one has $\tau=\frac{3}{2}$ and equation (77) expresses the number of self-consistent stochastic equations needed for treating the SOC behavior as a function of the exponent of the corresponding multiplicative noise:

$$
n=\frac{2}{1-a} .
$$

In accordance with Fig. 13c, a self-consistent mean-field treatment is possible if the number of relevant equations is larger than the minimum magnitude $n_{c}=2$. Approaches [5-7,16,19] represent examples of such considerations, where noise is supposed to have additive character $(a=0)$. Switching the multiplicative noise leads to an $a$-growth and non-contradicting representation of the SOC demands an increase in the number of self-consistent equations: for example, within the field scheme [20] related to directed percolation $\left(a=\frac{1}{2}\right)$, the mean-field approximation is applicable for dimensions larger 


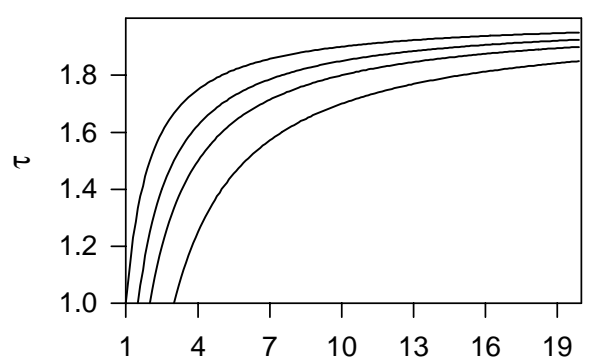

(a)

$n$

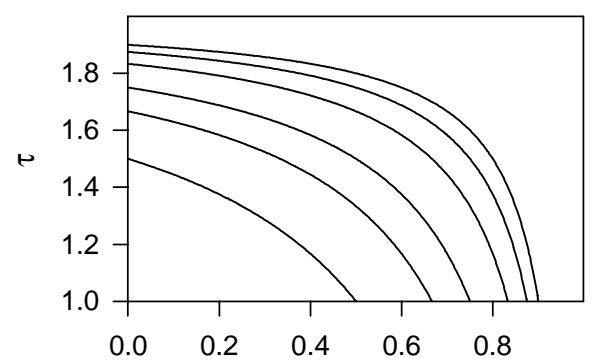

(b)

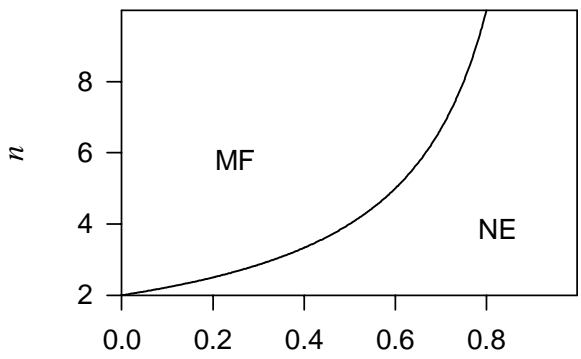

(c)

$a$

Fig. 13. Dependences of exponent $\tau$ : (a) on equations number $n$ ( $a=0, \frac{1}{3}, \frac{1}{2}, \frac{2}{3}$ from top to bottom); (b) on exponent $a$ ( $n=2,3,4,6,8,10$ from bottom to top); (c) phase diagram for mean-field and non-extensivity domains.

than the critical magnitude $d_{c}=4$; here and in Refs. [17,18] the Lorenz scheme $(n=3)$ with multiplicative noise is characterized by the exponent $a=\frac{1}{3}$ (see below).

Let us now focus on the relation of the above exponents to the non-extensivity parameter $q$ related to Tsallis definitions (57) and (58) [24]. The relevant kinetic behavior could be described by the nonlinear Fokker-Planck equation

$$
\mathscr{D}_{t}^{\omega} P(s, t)=\mathscr{D}_{-s}^{2} P^{q}(s, t),
$$

where $\mathscr{D}_{t}^{\omega}$ is the fractional derivative and the measure units are chosen in such a way that the effective diffusion coefficient disappears $(\omega>0, q>0$ are the relevant exponents $[33,34])$. Supposing a normalized distribution function in a self-similar form type of Eq. (2)

$$
P(s, t)=s_{c}^{-1} \mathscr{P}(x), \quad s_{c} \equiv s_{c}(t), \quad x \equiv s / s_{c},
$$

we obtain

$$
s_{c}^{q+1} \sim t^{\omega}, \quad \mathscr{P}^{q-1} \sim x^{2} .
$$

On the other hand, we could use the fractional Fokker-Planck equation of the type of Eq. (71):

$$
\mathscr{D}_{t}^{\omega} P(s, t)=\mathscr{D}_{-s}^{2 \pi} P(s, t) .
$$


Inserting the solution (80), one finds the dependencies

$$
s_{c}^{2 \varpi} \sim t^{\omega}, \quad \mathscr{P} \sim x^{2 \varpi-1}, \quad x \rightarrow 0
$$

whose comparison with Eqs. (81) yields

$$
1+q=2 \varpi .
$$

Because the average $|s|$ in Eq. (72) is reduced to the scale $s_{c}$ in the case of self-similar systems, the relevant dependencies (73), (81), and (83) give

$$
1+q=z \omega .
$$

Combining this equality with Eqs. (75) and (76) leads to the resulting expression for the non-extensivity parameter of the considered system:

$$
1+q=\frac{2}{n(1-a)} \text {. }
$$

The minimal magnitude $q=2 / n-1$ is related to systems with additive noise $(a=0)$, which is relevant to the mean-field picture at a number of governing equations $n<2$ $(n=1)$. Switching the multiplicative noise with increasing exponent $a>0$ leads to a $q$-growth and the self-organizing system gets a non-extensive character $(q \geqslant 1)$ in the limit $n \leqslant 1 /(1-a)$. In accordance with the above estimation the fractional Lorenz system is non-extensive, essentially if the exponent $a>\frac{2}{3}$.

It is worth to remember that above we have assumed the superdiffusion process only to be related to Lévy flights at discrete time instant with arbitrary displacements, including infinite ones [35]. Related to the Fokker-Planck equation (71), such processes are characterized by exponents $\omega=1$ and $\varpi<1$, the first of which is constant, whereas the second one characterizes the fractal time-sequence of the Lévy flights and leads to the dynamical exponent $z \equiv 2 \varpi / \omega<2$ (see the last of Eqs. (73)). The probability distribution of the displacement $\mathbf{x}$, that is dependent on microscopic conditions, reads

$$
p(\mathbf{x}) \sim x^{-(D+\gamma)}
$$

and is characterized by the fractal dimension $D$ and the microscopic step exponent $\gamma$. It is obvious that in the case of rare events, when $\gamma<2$, the dynamical exponent $z$ is reduced to the microscopic step exponent $(z=\gamma<2)$, whereas at $\gamma \geqslant 2$ one has $z=2[36]$.

In the opposite case of subdiffusion processes, a microscopical ingredient is the random Lévy walks instead of the discrete Lévy flights. This process evolves continuously in course of the time over discrete placed traps, so that the exponents $\omega<1$ describes fractal properties of this space that depend on microscopic conditions. These properties generate the transformation of the usual Boltzmann-Gibbs statistics in a non-extensive manner [24]. The subdiffusion process is presented by the Tsallis-type distribution [37]

$$
p(\mathbf{x}) \propto\left[1+\beta(q-1) x^{2}\right]^{-1 /(q-1)}, \quad \beta=\text { const }>0,
$$

where the deviation $q-1$ of the non-extensive parameter is caused by the fractal nature of the system phase space that is connected to the step exponent $\gamma$ as follows:

$$
q=1+\frac{2}{D+\gamma} \text {. }
$$


A formal advantage of this distribution (88) is that the corresponding $q$-weighted average

$$
\left\langle\mathbf{x}^{2}\right\rangle_{q} \equiv \int \mathbf{x}^{2} p^{q}(\mathbf{x}) \mathrm{d}^{D} x
$$

where the integrand varies as $x^{-(1+\gamma)}$, converges for arbitrary step exponents $\gamma>0$. As a result, the equation of motion of the random Lévy walker is given by

$$
\left\langle\mathbf{x}^{2}\right\rangle_{q} \sim t^{\omega}, \quad \omega= \begin{cases}q-1 & \text { at } \gamma, q<2, \\ 1-(q-1) \frac{D}{2} & \text { at } \gamma \geqslant 2, q>1 .\end{cases}
$$

In contrast to Eq. (83), where the exponents $\varpi<1, \omega=1$ are relevant for the superdiffusion, one here has inverted relations $\varpi=1, \omega<1$. Thus, in accordance with the subdiffusion nature, the last equality (73) yields the dynamical exponent $z>2$.

In the general case $\varpi, \omega \neq 1$, inserting Eqs. (91) into relation (85) leads to the result

$$
z= \begin{cases}\frac{q+1}{1-\frac{D}{2}(q-1)} & \text { at } 1<q \leqslant q_{D}, \\ \frac{q+1}{q-1} & \text { at } q_{D} \leqslant q \leqslant 2,\end{cases}
$$

where the boundary value of the non-extensivity parameter is introduced

$$
q_{D} \equiv \frac{4+D}{2+D} .
$$

To avoid a mistake, let us focus that in contrast to equalities (1), (74) and (75), which could be related to both the real phase space and the configurational one (the latter is spanned by variables of governing equations), the above obtained relations (91)-(93) are relevant for the real phase space only. This is reflected by addressing the fractal dimension $D$ to the only real coordinate space in the former case, whereas in the latter it is reduced to the effective value (76). According to our treatment, a central role is played by relation (76) since, in analogy with a renormalization group, we have considered the properties of the configurational space but not real diffusion process.

Finally, let us ask the question, why the Lorenz system is used and not the Rössler one or another? The reason can be recognized within a supersymmetry field approach, whose use shows that the Lorenz system could be generated by the Langevin equation for an order parameter that is relevant for a standard stochastic system [38]. On the other hand, it is easy to see with microscopic consideration that the Lorenz system is related to the simplest Hamiltonian of a boson-fermion system [39]. Here, the bosons are described by creation and annihilation operators $b_{l}^{+}, b_{l}$, satisfying the usual commutation relation: $\left[b_{l}, b_{m}^{+}\right]=\delta_{l m}$, where $l, m$ are the site numbers. The two-level Fermion subsystem is described by operators $a_{l \alpha}^{+}, a_{l \alpha}, \alpha=1,2$, that fulfill the anti-commutation relation $\left\{a_{l \alpha}, a_{m \beta}^{+}\right\}=\delta_{l m} \delta_{\alpha \beta}$. The occupation numbers $b_{\mathbf{k}}^{+} b_{\mathbf{k}}$ determine the Boson distribution in k-representation that corresponds to the Fourier transform over lattice sites $l$. To represent the Fermi subsystem we introduce the operator $d_{l} \equiv a_{l 1}^{+} a_{l 2}$ determining the polarization with respect to the saturation over levels $\alpha=1,2$, as well as the occupation numbers $n_{l \alpha} \equiv a_{l \alpha}^{+} a_{l \alpha}$. The behavior of the system is defined by the 
Dicke Hamiltonian $(\hbar=1)$

$$
H=\sum_{\mathbf{k}}\left\{\left(E_{1} n_{\mathbf{k} 1}+E_{2} n_{\mathbf{k} 2}\right)+\omega_{\mathbf{k}} b_{\mathbf{k}}^{+} b_{\mathbf{k}}+\frac{\mathrm{i}}{2} w\left(b_{\mathbf{k}}^{+} d_{\mathbf{k}}-d_{\mathbf{k}}^{+} b_{\mathbf{k}}\right)\right\},
$$

where $E_{1,2}$ are Fermi energies, $\omega_{\mathbf{k}}$ is the Boson dispersion law, the imaginary unit before the interaction $w$ reflects the Hermitian property.

The Heisenberg equations of motion are

$$
\begin{aligned}
& \dot{b}_{\mathbf{k}}=-\mathrm{i} \omega_{\mathbf{k}} b_{\mathbf{k}}+\frac{w}{2} d_{\mathbf{k}}, \\
& \dot{d}_{\mathbf{k}}=-\mathrm{i} \Delta d_{\mathbf{k}}+\frac{w}{2} b_{\mathbf{k}}\left(n_{\mathbf{k} 2}-n_{\mathbf{k} 1}\right), \quad \Delta \equiv E_{2}-E_{1}, \\
& \dot{n}_{\mathbf{k} 1}=\frac{w}{2}\left(b_{\mathbf{k}}^{+} d_{\mathbf{k}}+d_{\mathbf{k}}^{+} b_{\mathbf{k}}\right), \quad \dot{n}_{\mathbf{k} 2}=-\frac{w}{2}\left(b_{\mathbf{k}}^{+} d_{\mathbf{k}}+d_{\mathbf{k}}^{+} b_{\mathbf{k}}\right) .
\end{aligned}
$$

In resonance, the first terms on the right-hand sides of equations (96) and (97) containing frequencies $\omega_{\mathbf{k}}$ and $\Delta$ may be suppressed by introducing the multipliers $\exp \left(-\mathrm{i} \omega_{\mathbf{k}} t\right)$ and $\exp (-\mathrm{i} \Delta t)$ for the time dependencies $b_{\mathbf{k}}(t), d_{\mathbf{k}}(t)$, respectively. To take the dissipation into account, these frequencies acquire additional imaginary terms $-\mathrm{i} / \tau_{x},-\mathrm{i} / \tau_{y}$ characterized by relaxation times $\tau_{x}, \tau_{y}$ (here the conditions $\operatorname{Im} \omega_{\mathbf{k}}<0, \operatorname{Im} \Delta<0$ reflect the causality principle). As a result, equations (96) and (97) get the dissipative terms $-b_{\mathbf{k}} / \tau_{x},-d_{\mathbf{k}} / \tau_{y}$, where $\tau_{x}$ is the relaxation time of Boson distribution and $\tau_{y}$ is the Fermion polarization time. Obviously, one can suppose that the dissipation also influences the Fermi levels occupancies $n_{\mathbf{k} \alpha}(t)$. However, since the stationary values are $n_{\mathbf{k} \alpha}^{0} \neq 0$ (in case of an external drive $n_{\mathbf{k} 2}^{0}>n_{\mathbf{k} 1}^{0}$ ), the dissipative terms in Eqs. (97) take much more complicated form $-\left(n_{\mathbf{k} \alpha}-n_{\mathbf{k} \alpha}^{0}\right) / \tau_{S}$, where $\tau_{S}$ is the relaxation time of the Fermion distribution over levels $\alpha=1,2$.

Now, let us introduce the macroscopic quantities

$$
\begin{array}{ll}
u_{\mathbf{k}} \equiv\left\langle b_{\mathbf{k}}^{+}\right\rangle=\left\langle b_{\mathbf{k}}\right\rangle, & v_{\mathbf{k}} \equiv\left\langle d_{\mathbf{k}}\right\rangle=\left\langle d_{\mathbf{k}}^{+}\right\rangle, \\
S_{\mathbf{k}} \equiv\left\langle n_{\mathbf{k} 2}-n_{\mathbf{k} 1}\right\rangle, & S_{\mathbf{k}}^{0} \equiv\left\langle n_{\mathbf{k} 2}^{0}-n_{\mathbf{k} 1}^{0}\right\rangle,
\end{array}
$$

where the angular brackets denote thermodynamical averaging. Then, neglecting the correlation in particle distribution over quantum states and omitting the dependence on the wave vector $\mathbf{k}$, the Heisenberg equations (96)-(97) result in the initial Lorenz system (6), (8) and (9), with parameters $a=1, \tau_{x}=2 / w, D=(2 w)^{-1}$. The dimensionless variables in the system (10)-(12) are as follows: $u=w\left(\tau_{y} \tau_{S}\right)^{1 / 2} \dot{x}, v=w\left(\tau_{y} \tau_{S}\right)^{1 / 2} \dot{y}$ and $S=\left(w \tau_{y} / 2\right) y^{\prime}$.

As a result, we come to the conclusion that the Lorenz system is microscopically relevant for the simplest boson-fermion system defined by the Dicke Hamiltonian (94). At first glance, it can be shown that corresponding expression for a macroscopic (effective) Hamiltonian is a synergetic potential, whose dependence on the degrees of freedom $u$, $v, S$ can generate the Lorenz system. But such a dependence is absent because the effective Hamiltonian has to take into account quite different commutation rules for different freedom degrees. An obvious advantage of the above mentioned supersymmetry theory [38] and microscopic approach is that we have the explicit possibility to handle such a difference. This situation is generally relevant for known problem of the description of systems with intermediate statistics (see Ref. [40]). 


\section{Appendix A}

Here the basic properties of fractional integral and derivative, as well as Jackson derivative are quoted for convenience. The integral of fractional order $\varpi$ is defined by equality $[30,31]$

$$
\mathscr{I}_{x}^{\varpi} f(x) \equiv \frac{1}{\Gamma(\varpi)} \int_{0}^{x} \frac{f\left(x^{\prime}\right)}{\left(x-x^{\prime}\right)^{1-\varpi}} \mathrm{d} x^{\prime}, \quad \varpi>0,
$$

where $f(x)$ is an arbitrary function, $\Gamma(x)$ is the standard Gamma-function. To be inverted to the fractional integral, the relevant derivative $\mathscr{D}_{x}^{\varpi} \equiv \mathscr{I}_{x}^{-\varpi}$ of order $\varpi>0$ is determined as follows:

$$
\mathscr{D}_{x}^{\varpi} f(x) \equiv \frac{1}{\Gamma(-\varpi)} \int_{0}^{x} \frac{f\left(x^{\prime}\right)}{\left(x-x^{\prime}\right)^{1+\varpi}} \mathrm{d} x^{\prime} .
$$

In the region $0<\varpi<1$ it is convenient to use the expression

$$
\mathscr{D}_{x}^{\varpi} f(x) \equiv \frac{\varpi}{\Gamma(1-\varpi)} \int_{0}^{x} \frac{f(x)-f\left(x^{\prime}\right)}{\left(x-x^{\prime}\right)^{1+\varpi}} \mathrm{d} x^{\prime},
$$

where we take into account the known equality $x \Gamma(x)=\Gamma(x+1)$ for $x \equiv-\varpi$.

Let us introduce a Jackson $q$-derivative, whose advantage for analysis of self-similar system is that this derivative determines the rate of the variation of a function $f(x)$ with respect to the dilatation $q \neq 1$, but not to the shift $\mathrm{d} x \rightarrow 0$, as in usual case $q=1$. According to such a definition, the Jackson $q$-derivative reads

$$
\mathscr{D}_{q} f(x) \equiv \frac{f(q x)-f(x)}{q-1}, \quad q>0 .
$$

For the important case of a homogeneous function that satisfies to condition

$$
f(q x) \equiv q^{\alpha} f(x),
$$

where $q>0$ is a dilatation parameter and $\alpha>0$ is an exponent, the Jackson $q$-derivative is reduced to Jackson $q$-number

$$
\mathscr{D}_{q} f(x)=[\alpha]_{q} f(x), \quad[\alpha]_{q} \equiv \frac{q^{\alpha}-1}{q-1} .
$$

It is easy to see that $[\alpha]_{q} \rightarrow \alpha$ for $q \rightarrow 1$, and that $[\alpha]$ scales as $q^{\alpha-1}$ for $q \rightarrow \infty$. On the other hand, the Tsallis $q$-logarithmic function

$$
\ln _{q} x \equiv \frac{x^{q-1}-1}{q-1}
$$

can be represented in the form of the Jackson $q$-number (A.6) with the index $\alpha=$ $(q-1)(\ln x / \ln q)$. Accompanied by Eqs. (A.6) this relation and obvious equality

$$
\ln _{q}(x y)=\ln _{q} x+\ln _{q} y+(q-1)\left(\ln _{q} x\right)\left(\ln _{q} y\right)
$$

lead to the important rule for the Jackson derivative:

$$
\mathscr{D}_{q}[f(x) g(x)]=\left[\mathscr{D}_{q} f(x)\right] g(x)+f(x)\left[\mathscr{D}_{q} g(x)\right]+(q-1)\left[\mathscr{D}_{q} f(x)\right]\left[\mathscr{D}_{q} g(x)\right] .
$$


To generalize Eqs. (A.3) and (A.4), let us finally introduce a fractional $\varpi$-derivative:

$$
\mathscr{D}^{\varpi} f(x) \equiv \frac{\varpi x^{-\varpi}}{\Gamma(1-\varpi)} \int_{0}^{1} \frac{f(x)-f(q x)}{(1-q)^{1+\varpi}} \mathrm{d} q, \quad 0<\varpi<1 .
$$

In the case of a self-similar system, the function $f(x)$ is homogeneous, i.e., satisfies to condition (A.5). Then, the definition (A.10) is simplified:

$$
\mathscr{D}^{\varpi} f(x) \equiv\{\alpha\}_{\varpi} x^{-\varpi} f(x), \quad 0<\varpi<1,
$$

where the fractional $\varpi$-number is

$$
\{\alpha\}_{\varpi} \equiv \frac{\varpi}{\Gamma(1-\varpi)} \int_{0}^{1} \frac{1-q^{\alpha}}{(1-q)^{1+\varpi}} \mathrm{d} q, \quad 0<\alpha, \quad 0<\varpi<1 .
$$

It can be expressed in terms of $\Gamma$-functions:

$$
\{\alpha\}_{\varpi}=\frac{\Gamma(\alpha+1)}{\Gamma(\alpha+1-\varpi)}-\frac{1}{\Gamma(1-\varpi)} .
$$

This number increases monotonically with growth of both $\alpha, \varpi$, and has zero value at $\varpi=0, \alpha=0$ and characteristic values $\left\{\frac{1}{2}\right\}_{1 / 2}=\sqrt{\pi} / 2-1 / \sqrt{\pi} \simeq 0.322,\{1\}_{1}=1$. Such behavior is characterized by the particular dependencies

$$
\{\alpha\}_{\varpi}= \begin{cases}\alpha & \text { at } \varpi=1, \\ \Gamma(1+\alpha)-\frac{1}{\Gamma(1-\alpha)} & \text { at } \varpi=\alpha, \\ \frac{\varpi}{\Gamma(2-\varpi)} & \text { at } \alpha=1 .\end{cases}
$$

Thus, if the $q$-number (A.6) converges to an exponent $\alpha$ in the limit $q \rightarrow 1$, the $\varpi$-number (A.12) corresponding to the fractional integral (A.10) is reduced to a factor $\alpha$ at $\varpi=1$.

\section{References}

[1] P. Bak, How Nature Works: The Science of Self-Organized Criticality, Oxford University Press, Oxford, 1997.

[2] H.J. Jensen, Self-Organized criticality. Emergent complex behavior in physical and biological systems, in: Cambridge Lecture Notes in Physics, Cambridge University Press, Cambridge, 1998.

[3] M. Parzuski, S. Maslov, P. Bak, Phys. Rev. E 53 (1996) 414.

[4] S.F. Edwards, D.R. Wilkinson, Proc. R. Soc. A 381 (1982) 17.

[5] A. Mehta, G.C. Barker, Rep. Prog. Phys. 57 (1994) 383.

[6] J.-P. Bouchaud, M.E. Cates, J.R. Prakash, S.F. Edwards, J. Phys. I (France) 4 (1994) 1383.

[7] K.P. Hadeler, C. Kuttler, Granular Matter 2 (1999) 9.

[8] P. Bak, K. Sneppen, Phys. Rev. Lett. 71 (1993) 4083.

[9] T. Halpin-Healy, Y.-C. Zhang, Phys. Rep. 254 (1995) 215.

[10] D. Dhar, R. Ramaswamy, Phys. Rev. Let. 63 (1989) 1659;

D. Dhar, Phys. Rev. Let. 64 (1991) 1613.

[11] L. Pietronero, A. Vespignani, S. Zapperi, Phys. Rev. Lett. 72 (1994) 1690;

L. Pietronero, A. Vespignani, S. Zapperi, Phys. Rev. E 51 (1995) 1711.

[12] P. Bak, C. Tang, K. Wiesenfeld, Phys. Rev. Lett. 59 (1987) 381.

[13] L.P. Kadanoff, S.R. Nagel, L. Wu, S. Zhu, Phys. Rev. A 39 (1989) 6524.

[14] P. Grassberger, S.S. Manna, J. Phys. (France) 51 (1990) 1077. 
[15] T. Hwa, M. Kadar, Phys. Rev. A 45 (1992) 7002.

[16] L. Gil, D. Sornette, Phys. Rev. Lett. 76 (1996) 3991.

[17] A.I. Olemskoi, A.V. Khomenko, JETP 83 (1996) 1180.

[18] A.I. Olemskoi, A.V. Khomenko, Phys. Rev. E 63 (2001) 036116.

[19] A. Vespignani, S. Zapperi, Phys. Rev. Lett. 78 (1997) 4793;

A. Vespignani, S. Zapperi, Phys. Rev. E 57 (1998) 6345.

[20] A. Vespignani, R. Dickman, M.A. Muñoz, S. Zapperi, Phys. Rev. Lett. 81 (1998) 5676;

A. Vespignani, R. Dickman, M.A. Muñoz, S. Zapperi, Phys. Rev. E 62 (2000) 4564.

[21] J. Zinn-Justin, Quantum Field Theory and Critical Phenomena, Clarendon Press, Oxford, 1993.

[22] S.F. Edwards, R.B.S. Oakeshott, Physica A 157 (1989) 1080.

[23] S.F. Edwards, in: A. Metha (Ed.), Granular Matter: An Interdisciplinary Approach, Springer, New York, 1994.

[24] C. Tsallis, in: S. Abe, Y. Okamoto (Eds.), Lecture Notes in Physics, Springer, Heidelberg, 2001.

[25] G.M. Zaslavsky, Chaos 4 (1994) 25;

G.M. Zaslavsky, Physica D 76 (1994) 110;

A.I. Saichev, G.M. Zaslavsky, Chaos 7 (1997) 753.

[26] H. Risken, The Fokker-Planck Equation, Springer, Berlin, 1989.

[27] G. Haken, Synergetics, An Introduction, Springer, Berlin, 1983.

[28] A.I. Olemskoi, Physics-Uspekhi 41 (1998) 269.

[29] M. Cencini, M. Falcioni, E. Olbrich, H. Kantz, A. Vulpiani, Phys. Rev. E 62 (2000) 427.

[30] S.G. Samko, A.A. Kilbas, O.I. Marichev, Fractional Integrals and Derivatives-Theory and Applications, Gordon and Breach, New York, 1993.

[31] R. Hilfer (Ed.), Applications of Fractional Calculus in Physics, World Scientific, Singapore, 2000.

[32] A. Chessa, E. Marinari, A. Vespignani, S. Zapperi, Phys. Rev. E 57 (1998) R6241.

[33] A.I. Olemskoi, JETP Lett. 71 (2000) 285.

[34] A.I. Olemskoi, D.O. Kharchenko, Physica A 293 (2001) 178.

[35] J.-P. Bouchaud, A. Georges, Phys. Rep. 195 (1991) 127.

[36] H.C. Fogedby, Phys. Rev. E 58 (1998) 1690.

[37] D.H. Zanette, P.A. Alemany, Phys. Rev. Lett. 75 (1995) 366.

[38] A.I. Olemskoi, A.V. Khomenko, cond-mat/0008121.

[39] A.I. Olemskoi, Theory of Structure Transformations in Non-Equilibrium Condensed Matter, NOVA Science, New York, 1999.

[40] G. Kaniadakis, cond-mat/0103467. 